\title{
Mixed convection flow and heat transfer in a vertical wavy channel containing porous and fluid layer with traveling thermal waves
}

\author{
J.C. Umavathi* and M. Shekar \\ Department of Mathematics, Gulbarga University, Gulbarga-585 106, Karnataka, INDIA. \\ *Corresponding Author: jc_uma11@yhaoo.com.
}

\begin{abstract}
Mixed convection flow and heat transfer in a vertical wavy channel filled with porous and fluid layers is studied analytically. The flow in the porous medium is modeled using Darcy-Brinkman equation. The coupled non-linear partial differential equations describing the conservation of mass, momentum and energy are solved by linearization technique, wherein the flow is assumed to be in two parts; a mean part and a perturbed part. Exact solutions are obtained for the mean part and a perturbed part is solved using long wave approximation. Separate solutions are matched at the interface using suitable matching conditions. Results for a wide range of governing parameters such as Grashof number, viscosity ratio, width ratio, conductivity ratio, and traveling thermal temperature are plotted for different values of porous parameter on the velocity and temperature fields. Closed form expression for the skin friction and Nusselt number at both left and right channel walls are also derived and all the results are depicted pictorially. It is found that the presence of porous matrix, viscosity ratio and conductivity ratio suppress the velocity whereas, Grashof number and width ratio promotes the velocity parallel to the flow direction and reversal effect is observed on the velocity perpendicular to the flow direction.
\end{abstract}

Keywords: convective flow; wavy channel; porous medium; traveling thermal waves.

DOI: http://dx.doi.org/10.4314/ijest.v3i6.16

\section{Introduction}

Mixed convection heat transfer in porous media has received increasing interest over last twenty years, due to its numerous applications in geophysics and energy-related systems. Conservation in ducted flow may occur in many applications, such as heat exchangers, chemical processing equipment, transport of heated and cooled fluids, solar collectors and micro-electronic cooling. Buoyancy effects distort the velocity and temperature profiles relative to the forced convection case. This phenomenon is of substantial significance because it may strongly affect wall friction, pressure drop, and heat transfer, occurrence of extreme temperatures and stability of the flow. Convective heat transfer and fluid flow in a system containing simultaneously a fluid reservoir and a porous medium saturated with fluid is of great mathematical and physical interest. More specifically the existence of a fluid layer adjacent to layer of fluid saturated porous medium is a common occurrence in both geophysical and engineering environments. The fundamental nature and the growing volume of work in this area is amply documented in the books by Ingham and Pop (2005), Vafai (2005), Nield and Bejan (2006) etc.

Composite systems are part of numerous and other engineering applications also, such as fibrous and granular insulation, porous insulation of ducts, ambient air heat transfer from hair covered skin, grain storage, and drying paper. Freezing of soils and melting of ice frozen soils due to the change in weather conditions also require the knowledge of interaction mechanism between the fluid and porous layers. Composite layers are also find application in porous journal bearings.

Deajani et al. (1986) and Nield (1983) have studied the thermal instabilities of a superposed porous and fluid layer using Darcy's law together with matching conditions. Masuoka (1974) has observed convective flow in a layer of fluid heated from below and divided by a horizontal porous wall. He has found that the porous wall suppresses the convection. Recently, heat transfer in channels partially filled with porous media has received considerable attention and was the focus of several investigations (Chikh et al., 1995 and Vafai and Kim, 1995). As previously mentioned, the need for better understanding of heat 
transfer in porous media is motivated by numerous engineering applications encountered. In general, most analytical studies of fluid flow adopt Darcy's law (refer Nield and Bejan, 2006 for different models such as Darcy model, Darcy-Brinkman model and Brinkman-Forchheimer model). In the study conducted by Al-Nimr and Alkam (1998) there appears to be very limited research on the problems of forced convection in composite fluids and porous layers. Beavers and Joseph (1967) first investigated the fluid mechanics at the interface between fluid layer and a porous medium over a flat plate. Rudraiah (1985) investigated the same problem using Darcy-Brinkman model. Neild (1991) discussed the limitation of the Brinkman-Forchheimer model in porous media and the interface, between the clear fluid and porous region. Later on Vafai and Kim (1995) presented an exact solution for the same problem. Recently Tang, et al. (2010) studied the combined heat and moisture convective transport in a partial enclosure with multiple free ports.

Composite layer flows are exciting because of the modeling challenge that is thrown at researchers to model interface and boundary condition/s. By browsing the literature, it becomes evident that the interface conditions which have been in use are either of the 'slip' or 'stick' type of condition (see Beavers and Joseph, 1967 and Ochoa-Tapia and Whitaker, 1995). Several works have appeared on composite layer flows using these conditions or variant of them. Vafai and Kim (1990) presented an exact solution for the fluid flow at the interface between porous medium and a fluid layer including the inertia and boundary effect. They considered the shear stress in the fluid and the porous medium were taken to be equal at the interface region. Kuznetsov (1998) assumed that the shear stress jump is inversely proportional to the permeability of the porous medium. Later Alazmi and Vafai (2001) gave a detailed analysis of different types of interfacial conditions between a porous medium and a fluid layer which is the bench mark article to understand various types of interface conditions found in the literature. Following the analysis for interfacial conditions as defined in Vafai and Thiyagaraja (1987), Malashetty and his research group worked on flow and heat transfer through composite porous medium through channels. Convective flow and heat transfer in an inclined channel bounded by two rigid plates with one region filled with porous matrix saturated with a viscous fluid and another region with clear viscous fluid different from the fluid in the first region was studied by Malashetty et al. (2004). The same authors in 2005 analysed flow and heat transfer in an inclined channel consisting of a fluid layer sandwiched between two porous matrix layers. Oscillatory flow and heat transfer in composite porous medium channel was studied by Umavathi et al. (2006). An analysis of fully developed combined free and forced convective flow in a fluid saturated porous medium channel bounded by two vertical parallel plates was presented by Prathap Kumar et al. (2009). Recently Umavathi et al. (2010) found the exact solutions for the generalized plain Couette flow in a composite channel.

The dynamics of incompressible viscous fluid flows bounded by wavy walls is of special interest and has many practical applications in the transpiration cooling of re-entry vehicles and rocket boosters, cross-hatching on ablative surfaces and film vaporization in combustion chambers, the finishing of painted walls and in reducing friction of drag on the hulls of ships and submarines. Wavy walls are also employed in medical operations in order to increase mass transfer (blood oxy-genator Eldabe et al., 2008). Processes involving heat and mass transfer are often encountered in the chemical industry, in reservoir engineering connection with thermal recovery processes, and in the study of the dynamics of salty hot springs in the sea. In view of these applications, several authors have made investigations of the fluid flows over a wavy wall. Vajravelu and Sastri (1978) have made an interesting analysis of the free convective heat transfer in a viscous incompressible fluid bounded by a long (when compared to width of the channel) vertical wavy and parallel flat wall. Later Vajravelu (1989) studied the combined free and forced convection in hydromagnetic flows in a vertical wavy channel with traveling thermal waves. Malashetty et al. (2001) studied on magneto convective flow and heat transfer between vertical wavy wall and a parallel flat wall. Luo (2008) studied the flow of two superposed viscous fluid layers in a two-dimensional channel confined between a plane and a wavy wall by analytical and numerical methods at arbitrary Reynolds numbers. Srinivas and Muthuraj (2010) studied MHD flow with slip effects and temperature-dependent heat source in a vertical wavy porous space. Recently Umavathi et al. $(2010,2011)$ studied the flow and heat transfer in a long vertical channel composed of a smooth and a corrugated wall filled with two immiscible viscous fluids.

Much attention has not been given to the mixed convection flow and heat transfer in a fluid superposed porous medium in vertical wavy channel even though the study is useful in many areas of applications as mentioned above. Thus the objective of this work is to study the flow and heat transfer in a vertical wavy channel, containing porous layer saturated with a fluid and a clear viscous fluid layer. In this study, the porous matrix is assumed to be sparse; the Darcy-Brinkman model is thus adopted to describe the fluid flow in porous medium region.

\section{Mathematical formulation of the problem}

The geometry under consideration illustrated in Figure 1 consists of wavy walls in which $X$-axis is taken vertically upward, and parallel to the direction of buoyancy, and the $Y$-axis is normal to it. The wavy walls are represented by $Y=-h^{(1)}+a \cos (\lambda X+\theta)$ (right wavy wall) and $Y=h^{(2)}+a \cos (\lambda X)$ (left wavy wall). Considering different and constant temperatures $\hat{T}_{1}$ (right wavy wall)

and $\hat{T}_{2}$ (left wavy wall), where $\hat{T}_{2}>\hat{T}_{1}$. The region $-h^{(1)} \leq Y \leq 0$ is occupied by a fluid-saturated porous medium of density $\rho$, specific heat at constant pressure $C_{p}$, viscosity $\mu$, permeability $\kappa$, thermal conductivity $K$, thermal expansion coefficient $\beta$, and the region $0 \leq Y \leq h^{(2)}$ (region-II) is occupied by the fluid as in region-I without porous matrix.

We make the following assumptions: 
(a) the fluid properties are assumed to be constant and the Boussinesq approximation will be used so that the density variation is retained only in the buoyancy term;

(b) the flow is laminar and two-dimensional (that is, the flow is identical in vertical layers, which is a valid assumption);

(c) the wave length of the wavy wall which is proportional to $a^{-1}$ is very large where $a$ is the amplitude.
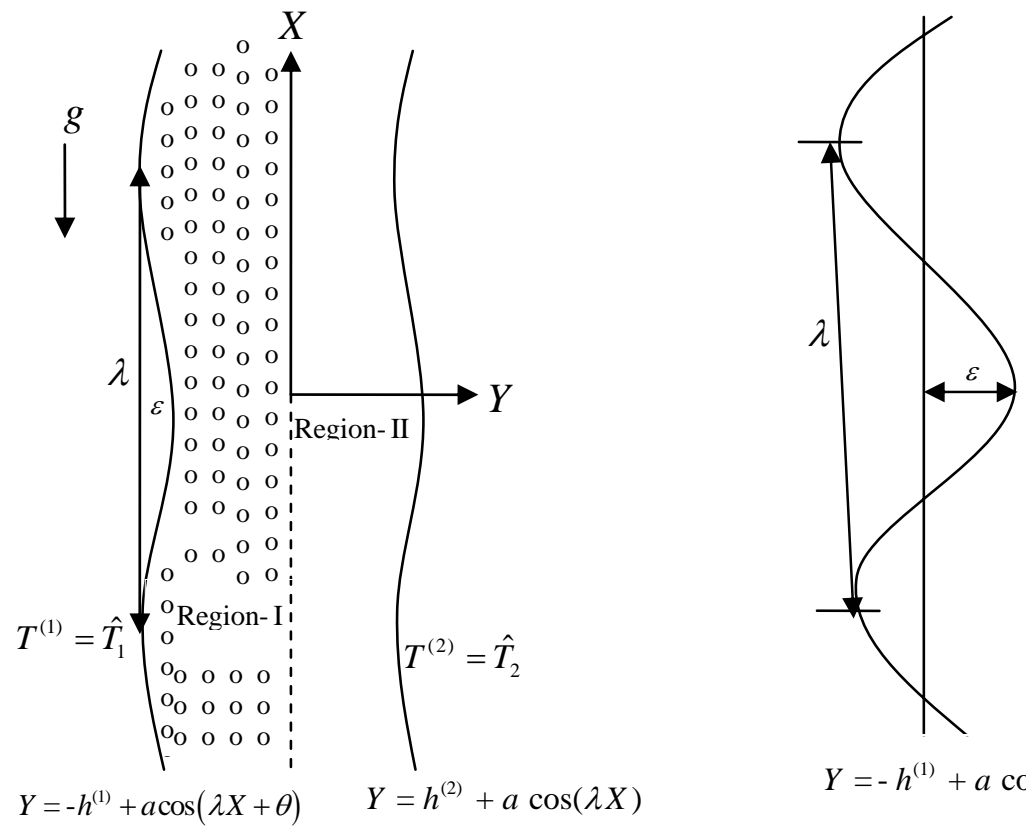

$$
Y=-h^{(1)}+a \cos (\lambda X+\theta)
$$

Figure 1: Physical configuration.

We consider fluid to be incompressible and the flow is steady and fully developed. Thus, with these the continuity equation, momentum equation, energy equation and state equation using Darcy-Brinkman model yield (Nield and Bejan, 2006).

Region - I

$$
\begin{gathered}
\frac{\partial U^{(1)}}{\partial X^{(1)}}+\frac{\partial V^{(1)}}{\partial Y^{(1)}}=0 \\
\rho\left(U^{(1)} \frac{\partial U^{(1)}}{\partial X^{(1)}}+V^{(1)} \frac{\partial U^{(1)}}{\partial Y^{(1)}}\right)=-\frac{\partial P^{(1)}}{\partial X^{(1)}}+\mu_{e f f}\left(\frac{\partial^{2} U}{\partial X^{2}}+\frac{\partial^{2} U}{\partial Y^{2}}\right)^{(1)}-\rho g-\frac{\mu}{\kappa} U^{(1)} \\
\rho\left(U^{(1)} \frac{\partial V^{(1)}}{\partial X^{(1)}}+V^{(1)} \frac{\partial V^{(1)}}{\partial Y^{(1)}}\right)=-\frac{\partial P^{(1)}}{\partial Y^{(1)}}+\mu_{e f f}\left(\frac{\partial^{2} V}{\partial X^{2}}+\frac{\partial^{2} V}{\partial Y^{2}}\right)^{(1)}-\frac{\mu}{\kappa} V^{(1)} \\
\rho C_{p}\left(U^{(1)} \frac{\partial T^{(1)}}{\partial X^{(1)}}+V^{(1)} \frac{\partial T^{(1)}}{\partial Y^{(1)}}\right)=K_{e f f}\left(\frac{\partial^{2} T}{\partial X^{2}}+\frac{\partial^{2} T}{\partial Y^{2}}\right)^{(1)} \\
\rho=\rho_{s}\left(1-\beta\left(T^{(1)}-T_{s}\right)\right)
\end{gathered}
$$

Region - II

$$
\begin{gathered}
\frac{\partial U^{(2)}}{\partial X^{(2)}}+\frac{\partial V^{(2)}}{\partial Y^{(2)}}=0 \\
\rho\left(U^{(2)} \frac{\partial U^{(2)}}{\partial X^{(2)}}+V^{(2)} \frac{\partial U^{(2)}}{\partial Y^{(2)}}\right)=-\frac{\partial P^{(2)}}{\partial X^{(2)}}+\mu\left(\frac{\partial^{2} U}{\partial X^{2}}+\frac{\partial^{2} U}{\partial Y^{2}}\right)^{(2)}-\rho g \\
\rho\left(U^{(2)} \frac{\partial V^{(2)}}{\partial X^{(2)}}+V^{(2)} \frac{\partial V^{(2)}}{\partial Y^{(2)}}\right)=-\frac{\partial P^{(2)}}{\partial Y^{(2)}}+\mu\left(\frac{\partial^{2} V}{\partial X^{2}}+\frac{\partial^{2} V}{\partial Y^{2}}\right)^{(2)}
\end{gathered}
$$




$$
\begin{gathered}
\rho C_{p}\left(U^{(2)} \frac{\partial T^{(2)}}{\partial X^{(2)}}+V^{(2)} \frac{\partial T^{(2)}}{\partial Y^{(2)}}\right)=K\left(\frac{\partial^{2} T}{\partial X^{2}}+\frac{\partial^{2} T}{\partial Y^{2}}\right)^{(2)} \\
\rho=\rho_{s}\left(1-\beta\left(T^{(2)}-T_{s}\right)\right)
\end{gathered}
$$

The fluid viscosity and the effective viscosity in the Brinkman term are distinguished respectively as $\mu$ and $\mu_{\text {eff }}$ in Eqs. (2) and (3). Most works which used the Brinkman model assumed that $\mu_{\text {eff }}=\mu$. However, recent direct numerical simulation (Martys et al., 1994) and recent experimental investigation (Givler and Altobelli, 1994) have demonstrated that there are situations when it is important to distinguish between these two coefficients. For example, in Givler and Altobelli (1994) a water flow through a tube filled with an open-cell rigid form of high porosity was investigated. It was obtained that for this flow $\mu_{\text {eff }}=\left(7.5_{-2.4}^{+3.4}\right) \mu$.

The boundary conditions on $U^{(j)}, V^{(j)}$ are both no-slip conditions and boundary conditions on $T$ are $\hat{T}_{1}$ at the left wall and $\hat{T}_{2}$ at the right wall. For the problem displayed in Figure 1 at the interface (between region-I and region-II) we utilize the assumption of Kim and Choi (1996) and Kuznetsov (1999) that is, continuity of velocity, continuity of shear stress, continuity of pressure gradient along the flow direction, continuity of temperature and continuity of heat flux and are given below

The relevant boundary and interface conditions on velocity are

$$
\begin{aligned}
U^{(1)}=V^{(1)}=0 \quad \text { at } Y=-h^{(1)}+a \cos (\lambda X+\theta) ; \quad U^{(2)}=V^{(2)}=0 \quad \text { at } Y=h^{(2)}+a \cos (\lambda X) \\
U^{(1)}=U^{(2)}, \quad V^{(1)}=V^{(2)}, \quad \mu_{\text {eff }}\left(\frac{\partial U}{\partial Y}+\frac{\partial V}{\partial X}\right)^{(1)}=\mu\left(\frac{\partial U}{\partial Y}+\frac{\partial V}{\partial X}\right)^{(2)}, \quad \frac{\partial P^{(1)}}{\partial X^{(1)}}=\frac{\partial P^{(2)}}{\partial X^{(2)}} \text { at } Y=0
\end{aligned}
$$

The relevant boundary and interface conditions for temperature are

$$
\begin{aligned}
& T^{(1)}=T_{1}[1+a \cos (\lambda X)] \\
&=\hat{T}_{1} \text { (say) } \quad \text { at } Y=-h^{(1)}+a \cos (\lambda X+\theta) \\
& T^{(2)}=T_{2}[1+a \cos (\lambda X)] \\
&=\hat{T}_{2}(\text { say }) \quad \text { at } Y=h^{(2)}+a \cos (\lambda X) \\
& T^{(1)}=T^{(2)}, K_{\text {eff }}\left(\frac{\partial T}{\partial Y}+\frac{\partial T}{\partial X}\right)^{(1)}=K\left(\frac{\partial T}{\partial Y}+\frac{\partial T}{\partial X}\right)^{(2)} \quad \text { at } Y=0
\end{aligned}
$$

We next introduce the non-dimensional flow variables as

$$
\begin{gathered}
x^{(1)}=\frac{X^{(1)}}{h^{(1)}}, x^{(2)}=\frac{X^{(2)}}{h^{(2)}}, y^{(1)}=\frac{Y^{(1)}}{h^{(1)}}, y^{(2)}=\frac{Y^{(2)}}{h^{(2)}}, u^{(1)}=\frac{h^{(1)}}{v} U^{(1)}, v^{(1)}=\frac{h^{(1)}}{v} V^{(1)}, u^{(2)}=\frac{h^{(2)}}{v} U^{(2)}, v^{(2)}=\frac{h^{(2)}}{v} V^{(2)}, \\
p^{(1)}=\frac{\rho P^{(1)}}{\mu^{2} / h^{(1)^{2}}}, p^{(2)}=\frac{\rho P^{(2)}}{\mu^{2} / h^{(2)^{2}}}, T^{*(1)}=\frac{T^{(1)}-T_{s}}{\hat{T}_{2}-\hat{T}_{1}}, T^{*(2)}=\frac{T^{(2)}-T_{s}}{\hat{T}_{2}-\hat{T}_{1}}, G r=\frac{h^{(1)^{3}} g \beta\left(\hat{T}_{2}-\hat{T}_{1}\right)}{v^{2}}, m=\frac{\mu_{e f f}}{\mu}, k=\frac{K_{e f f}}{K}, h=\frac{h^{(2)}}{h^{(1)}} \\
\sigma=\frac{h^{(1)}}{\sqrt{\kappa}}, \varepsilon=\frac{a}{h^{(1)}}, \lambda^{*}=\frac{\lambda}{h^{(1)}}, \operatorname{Pr}=\frac{C_{p} \mu}{K}
\end{gathered}
$$

In terms of these non-dimensional variables, the basic Eqs. (1) to (8) can be expressed in the dimensionless form, as, (for simplicity, the notation is considered as $x^{(1)}=x ; y^{(1)}=y$ in region-I and $x^{(2)}=x ; y^{(2)}=y$ in region-II)

Region-I

$$
\begin{gathered}
\frac{\partial u^{(1)}}{\partial x}+\frac{\partial v^{(1)}}{\partial y}=0 \\
u^{(1)} \frac{\partial u^{(1)}}{\partial x}+v^{(1)} \frac{\partial u^{(1)}}{\partial y}=-\frac{\partial p^{(1)}}{\partial x}+m\left(\frac{\partial^{2} u^{(1)}}{\partial x^{2}}+\frac{\partial^{2} u^{(1)}}{\partial y^{2}}\right)+G r T^{*(1)}-\sigma^{2} u^{(1)} \\
u^{(1)} \frac{\partial v^{(1)}}{\partial x}+v^{(1)} \frac{\partial v^{(1)}}{\partial y}=-\frac{\partial p^{(1)}}{\partial y}+m\left(\frac{\partial^{2} v^{(1)}}{\partial x^{2}}+\frac{\partial^{2} v^{(1)}}{\partial y^{2}}\right)-\sigma^{2} v^{(1)} \\
u^{(1)} \frac{\partial T^{*(1)}}{\partial y}+v^{(1)} \frac{\partial T^{*(1)}}{\partial y}=\frac{k}{\operatorname{Pr}}\left(\frac{\partial^{2} T^{*(1)}}{\partial x^{2}}+\frac{\partial^{2} T^{*(1)}}{\partial y^{2}}\right)
\end{gathered}
$$

Region-II

$$
\frac{\partial u^{(2)}}{\partial x}+\frac{\partial v^{(2)}}{\partial y}=0
$$




$$
\begin{gathered}
u^{(2)} \frac{\partial u^{(2)}}{\partial x}+v^{(2)} \frac{\partial u^{(2)}}{\partial y}=-\frac{\partial p^{(2)}}{\partial x}+\frac{\partial^{2} u^{(2)}}{\partial x^{2}}+\frac{\partial^{2} u^{(2)}}{\partial y^{2}}+G r h^{3} T^{*(2)} \\
u^{(2)} \frac{\partial v^{(2)}}{\partial x}+v^{(2)} \frac{\partial v^{(2)}}{\partial y}=-\frac{\partial p^{(2)}}{\partial y}+\frac{\partial^{2} v^{(2)}}{\partial x^{2}}+\frac{\partial^{2} v^{(2)}}{\partial y^{2}} \\
u^{(2)} \frac{\partial T^{*(2)}}{\partial x}+v^{(2)} \frac{\partial T^{*(2)}}{\partial y}=\frac{1}{\operatorname{Pr}}\left(\frac{\partial^{2} T^{*(2)}}{\partial x^{2}}+\frac{\partial^{2} T^{*(2)}}{\partial y^{2}}\right)
\end{gathered}
$$

Using Eqn. (11) boundary and interface conditions Eqn. (9) for velocity field become

$$
\begin{array}{r}
u^{(1)}=v^{(1)}=0 \text { at } \quad y=-1+\varepsilon \cos \left(\lambda^{*} x+\theta\right) ; \quad u^{(2)}=v^{(2)}=0 \text { at } \quad y=1+\varepsilon \cos \left(\lambda^{*} x\right) \\
u^{(1)}=\frac{u^{(2)}}{h}, v^{(1)}=\frac{v^{(2)}}{h}, \frac{\partial u^{(1)}}{\partial y}+\frac{\partial v^{(1)}}{\partial x}=\frac{1}{m h^{2}}\left(\frac{\partial u^{(2)}}{\partial y}+\frac{\partial v^{(2)}}{\partial x}\right), \frac{\partial p^{(1)}}{\partial x}=\frac{1}{h^{3}} \frac{\partial p^{(2)}}{\partial x} \text { at } y=0
\end{array}
$$

Using Eqn. (11) boundary and interface conditions Eqn. (10) for temperature field become

$$
\begin{gathered}
T^{*(1)}=0 \text { at } y=-1+\varepsilon \cos \left(\lambda^{*} x+\theta\right) ; \quad T^{*(2)}=1 \text { at } y=1+\varepsilon \cos \left(\lambda^{*} x\right) \\
T^{*(1)}=T^{*(2)}, \frac{\partial T^{*(1)}}{\partial y}+\frac{\partial T^{*(1)}}{\partial x}=\frac{1}{k h}\left(\frac{\partial T^{*(2)}}{\partial y}+\frac{\partial T^{*(2)}}{\partial x}\right) \text { at } y=0
\end{gathered}
$$

In the static fluid we have (see Vajravelu and Sastri, 1978)

$$
0=-\frac{\partial p_{s}}{\partial x}-\frac{\rho_{s} g h^{(1)^{3}}}{\rho v^{2}}=-\frac{\partial p_{s}}{\partial x}-\frac{\rho_{s} g h^{(2)^{3}}}{\rho v^{2}}
$$

In view of Eqn. (22). Equations (13) and (17) becomes

$$
\begin{gathered}
u^{(1)} \frac{\partial u^{(1)}}{\partial x}+v^{(1)} \frac{\partial u^{(1)}}{\partial y}=-\frac{\partial\left(p^{(1)}-p_{s}\right)}{\partial x}+m\left(\frac{\partial^{2} u^{(1)}}{\partial x^{2}}+\frac{\partial^{2} u^{(1)}}{\partial y^{2}}\right)-\sigma^{2} u^{(1)}+G r T^{*(1)} \\
u^{(2)} \frac{\partial u^{(2)}}{\partial x}+v^{(2)} \frac{\partial u^{(2)}}{\partial y}=-\frac{\partial\left(p^{(2)}-p_{s}\right)}{\partial x}+\frac{\partial^{2} u^{(2)}}{\partial x^{2}}+\frac{\partial^{2} u^{(2)}}{\partial y^{2}}+G r h^{3} T^{*(2)}
\end{gathered}
$$

\section{Solutions to the problem.}

Equations (12), (14)-(16), (18), (19), (23), and (24) are coupled nonlinear and are to be solved simultaneously. Due to the nonlinearity, analytical solutions are difficult; however approximate solutions can be obtained using perturbation techniques. Assuming that the solutions consists of a mean part and a perturbed part, velocity, pressure and temperature can be written as,

$$
\begin{gathered}
u^{(j)}(x, y)=u_{0}^{(j)}(y)+u_{1}^{(j)}(x, y) \\
v^{(j)}(x, y)=v_{1}^{(j)}(x, y) \\
p^{(j)}(x, y)=p_{0}^{(j)}(x, y)+p_{1}^{(j)}(x, y) \\
T^{*(j)}(x, y)=T_{0}^{*(j)}(y)+T_{1}^{*(j)}(x, y)
\end{gathered}
$$

where the perturbed quantities $u_{1}, v_{1}, p_{1}$ and $T_{1}^{*}$ are small compared with the mean or zeroth order quantities $u_{0}, T_{0}^{*}$. The asterisk on $T$ and $\lambda$ is removed for the sake of simplicity in the following process.

Using Eqs. (25) to (28) in the Eqs. (12), (14)-(16), (18), (19), (23), and (24), separating the mean part (zeroth order) and the perturbed part (first order), gives the following equations.

Zeroth order equations

$$
\begin{gathered}
\frac{d^{2} T_{0}^{(1)}}{d y^{2}}=0 \\
m \frac{d^{2} u_{0}^{(1)}}{d y^{2}}-\sigma^{2} u_{0}^{(1)}+G r T_{0}^{(1)}=0 \\
\frac{d^{2} T_{0}^{(2)}}{d y^{2}}=0 \\
\frac{d^{2} u_{0}^{(2)}}{d y^{2}}+G r h^{3} T_{0}^{(2)}=0
\end{gathered}
$$


First order equations

$$
\begin{gathered}
u_{0}^{(1)} \frac{\partial u_{1}^{(1)}}{\partial x}+v_{1}^{(1)} \frac{d u_{0}^{(1)}}{d y}=-\frac{\partial p_{1}^{(1)}}{\partial x}+m\left(\frac{\partial^{2} u_{1}^{(1)}}{\partial x^{2}}+\frac{\partial^{2} u_{1}^{(1)}}{\partial y^{2}}\right)-\sigma^{2} u_{1}^{(1)}+G r T_{1}^{(1)} \\
u_{0}^{(1)} \frac{\partial v_{1}^{(1)}}{\partial x}=-\frac{\partial p_{1}^{(1)}}{\partial y}+m\left(\frac{\partial^{2} v_{1}^{(1)}}{\partial x^{2}}+\frac{\partial^{2} v_{1}^{(1)}}{\partial y^{2}}\right)-\sigma^{2} v_{1}^{(1)} \\
u_{0}^{(1)} \frac{\partial T_{1}^{(1)}}{\partial x}+v_{1}^{(1)} \frac{d T_{0}^{(1)}}{d y}=\frac{k}{\operatorname{Pr}}\left(\frac{\partial^{2} T_{1}^{(1)}}{\partial x^{2}}+\frac{\partial^{2} T_{1}^{(1)}}{\partial y^{2}}\right) \\
u_{0}^{(2)} \frac{\partial u_{1}^{(2)}}{\partial x}+v_{1}^{(2)} \frac{d u_{0}^{(2)}}{d y}=-\frac{\partial p_{1}^{(2)}}{\partial x}+\frac{\partial^{2} u_{1}^{(2)}}{\partial x^{2}}+\frac{\partial^{2} u_{1}^{(2)}}{\partial y^{2}}+G r h^{3} T_{1}^{(2)} \\
u_{0}^{(2)} \frac{\partial v_{1}^{(2)}}{\partial x}=-\frac{\partial p_{1}^{(2)}}{\partial y}+\frac{\partial^{2} v_{1}^{(2)}}{\partial x^{2}}+\frac{\partial^{2} v_{1}^{(2)}}{\partial y^{2}} \\
u_{0}^{(2)} \frac{\partial T_{1}^{(2)}}{\partial x}+v_{1}^{(1)} \frac{d T_{0}^{(2)}}{d y}=\frac{1}{\operatorname{Pr}}\left(\frac{\partial^{2} T_{1}^{(2)}}{\partial x^{2}}+\frac{\partial^{2} T_{1}^{(2)}}{\partial y^{2}}\right)
\end{gathered}
$$

In view of Eqs. (25) to (28) the boundary and interface conditions as defined in Eqs. (20) and (21) can be split as follows, Zeroth order boundary and interface conditions for velocity and temperature are

$$
\begin{array}{lll}
u_{0}^{(1)}=0 \text { at } y=-1 ; & u_{0}^{(2)}=0 \text { at } y=1 ; & u_{0}^{(1)}=\frac{u_{0}^{(2)}}{h}, \frac{d u_{0}^{(1)}}{d y}=\frac{1}{m h^{2}} \frac{d u_{0}^{(2)}}{d y} \text { at } y=0 \\
T_{0}^{(1)}=0 \text { at } y=-1 ; & T_{0}^{(2)}=1 \text { at } y=1 ; & T_{0}^{(1)}=T_{0}^{(2)}, \frac{d T_{0}^{(1)}}{d y}=\frac{1}{k h} \frac{d T_{0}^{(2)}}{d y} \text { at } y=0
\end{array}
$$

First order boundary and interface conditions for velocity and temperature are

$$
\begin{gathered}
u_{1}^{(1)}=-\cos (\lambda x+\theta) \frac{d u_{0}^{(1)}}{d y}, v_{1}^{(1)}=0 \quad \text { at } y=-1 ; \quad u_{1}^{(2)}=-\frac{\cos (\lambda x)}{h} \frac{d u_{0}^{(2)}}{d y}, v_{1}^{(2)}=0 \quad \text { at } y=1 \\
u_{1}^{(1)}=\frac{1}{h} u_{1}^{(2)}, v_{1}^{(1)}=\frac{1}{h} v_{1}^{(2)}, \frac{\partial u_{1}^{(1)}}{\partial y}+\frac{\partial v_{1}^{(1)}}{\partial x}=\frac{1}{m h^{2}}\left(\frac{\partial u_{1}^{(2)}}{\partial y}+\frac{\partial v_{1}^{(2)}}{\partial x}\right), \frac{\partial p^{(1)}}{\partial x}=\frac{1}{h^{3}} \frac{\partial p^{(2)}}{\partial x} \quad \text { at } y=0 \\
T_{1}^{(1)}=-\cos (\lambda x+\theta) \frac{d T_{0}^{(1)}}{d y} \text { at } y=-1 ; \quad T_{1}^{(2)}=-\frac{\cos (\lambda x)}{h} \frac{d T_{0}^{(2)}}{d y} \quad \text { at } y=1 \\
T_{1}^{(1)}=T_{1}^{(2)}, \frac{\partial T_{1}^{(1)}}{\partial y}+\frac{\partial T_{1}^{(1)}}{\partial x}=\frac{1}{k h}\left(\frac{\partial T_{1}^{(2)}}{\partial y}+\frac{\partial T_{1}^{(2)}}{\partial x}\right) \quad \text { at } y=0
\end{gathered}
$$

In order to solve Eqs. (33) to (38), for the first order quantities it is convenient to introduce stream function $\bar{\psi}$ in the following form

$$
u_{1}^{(j)}=-\frac{\partial \bar{\psi}^{(j)}}{\partial y} \text { and } v_{1}^{(j)}=\frac{\partial \bar{\psi}^{(j)}}{\partial x} \quad \text { for } j=1,2
$$

The stream function approach reduces the number of dependent variables to be solved and also eliminates pressure from the list of variables. Differentiate Eqn. (33) with respect to $y$ and differentiate Eqn. (34) with respect to $x$ and then subtract Eqn. (33) with Eqn. (34) which will result in the elimination of pressure $p_{1}^{(1)}$. Similar procedure is opted for elimination of pressure $p_{1}^{(2)}$ from Eqs. (36) and (37). Equations (33) to (38) after elimination of $p_{1}^{(1)}$ and $p_{1}^{(2)}$, can be expressed in terms of the stream function $\bar{\psi}$ in the form

Region-I

$$
\begin{gathered}
u_{0}^{(1)} \bar{\psi}_{x y y}^{(1)}-\bar{\psi}_{x}^{(1)} u_{0 y y}^{(1)}+u_{0}^{(1)} \bar{\psi}_{x x x}^{(1)}-m\left(\bar{\psi}_{x x x x}^{(1)}+\bar{\psi}_{y y y y}^{(1)}\right)+\sigma^{2}\left(\bar{\psi}_{x x}^{(1)}+\bar{\psi}_{y y}^{(1)}\right)-2 \bar{\psi}_{x x y y}^{(1)}+G r T_{1 y}^{(1)}=0 \\
u_{0}^{(1)} T_{1 x}^{(1)}+\bar{\psi}_{x}^{(1)} T_{0 y}^{(1)}=\frac{k}{\operatorname{Pr}}\left(T_{1 x x}^{(1)}+T_{1 y y}^{(1)}\right)
\end{gathered}
$$

Region-II

$$
\begin{gathered}
u_{0}^{(2)} \bar{\psi}_{x y y}^{(2)}-\bar{\psi}_{x}^{(2)} u_{0 y y}^{(2)}+u_{0}^{(2)} \bar{\psi}_{x x x}^{(2)}-\bar{\psi}_{y y y y}^{(2)}-\bar{\psi}_{x x x x}^{(2)}-2 \bar{\psi}_{x x y y}^{(2)}+G r h^{3} T_{1 y}^{(2)}=0 \\
u_{0}^{(2)} T_{1 x}^{(2)}+\bar{\psi}_{x}^{(2)} T_{0 y}^{(2)}=\frac{1}{\operatorname{Pr}}\left(T_{1 x x}^{(2)}+T_{1 y y}^{(2)}\right)
\end{gathered}
$$


where a suffix $x$ or $y$ represents derivative with respect to $x$ or $y$ and 0 or 1 represents the zeroth order or first order terms respectively.

The corresponding boundary and interface conditions on velocity and temperature reduces to

$$
\begin{gathered}
\bar{\psi}_{y}^{(1)}=\cos (\lambda x+\theta) u_{0 y}^{(1)}, \bar{\psi}_{x}^{(1)}=0 \text { at } y=-1 ; \quad \bar{\psi}_{y}^{(2)}=\frac{\cos (\lambda x)}{h} u_{0 y}^{(2)}, \bar{\psi}_{x}^{(2)}=0 \text { at } y=1, \\
\bar{\psi}_{y}^{(1)}=\frac{\bar{\psi}_{y}^{(2)}}{h}, \bar{\psi}_{x}^{(1)}=\frac{\bar{\psi}_{x}^{(2)}}{h}, \bar{\psi}_{x x}^{(1)}-\bar{\psi}_{y y}^{(1)}=\frac{1}{m h^{2}}\left(\bar{\psi}_{x x}^{(2)}-\bar{\psi}_{y y}^{(2)}\right) \text { at } \quad y=0 \\
\bar{\psi}_{x}^{(1)} u_{0 y}^{(1)}-u_{0}^{(1)} \bar{\psi}_{x y}^{(1)}+m\left(\bar{\psi}_{x x y}^{(1)}+\bar{\psi}_{y y y}^{(1)}\right)-\sigma^{2} \bar{\psi}_{y}^{(1)}-G r T_{1}^{(1)}=\frac{1}{h^{3}}\left(\bar{\psi}_{x}^{(2)} u_{0 y}^{(2)}-u_{0}^{(2)} \bar{\psi}_{x y}^{(2)}+\bar{\psi}_{x x y}^{(1)}+\bar{\psi}_{y y y}^{(1)}-G r h^{3} T_{1}^{(2)}\right) \text { at } y=0 \\
T_{1}^{(1)}=-\cos (\lambda x+\theta) \frac{d T_{0}^{(1)}}{d y} \text { at } y=-1 ; \quad T_{1}^{(2)}=-\frac{\cos (\lambda x)}{h} \frac{d T_{0}^{(2)}}{d y} \text { at } y=1 \\
T_{1}^{(1)}=T_{1}^{(2)}, T_{1 x x}^{(1)}+T_{1 y y}^{(1)}=\frac{T_{1 x x}^{(2)}+T_{1 y y}^{(2)}}{k h} \text { at } y=0
\end{gathered}
$$

We assume stream function and temperature in the following form

$$
\bar{\psi}^{(j)}=\varepsilon e^{i \lambda x} \psi(y), T_{1}^{(j)}=\varepsilon e^{i \lambda x} t(y) \quad \text { for } j=1,2
$$

from which we infer

$$
u_{1}(x, y)=\varepsilon e^{i \lambda x} u_{1}(y), \quad v_{1}(x, y)=\varepsilon e^{i \lambda x} v_{1}(y)
$$

where $i$ is the imaginary unit.

In view of Eqn. (50), Eqs. (44) to (47) become

Region-I

Region-II

$$
\begin{gathered}
m \psi_{y y y y}^{(1)}-i\left(\lambda u_{0}^{(1)}+2 m \lambda^{2}+\sigma^{2}\right) \psi_{y y}^{(1)}+\left(i \lambda u_{0 y y}^{(1)}+\sigma^{2} \lambda^{2}+i \lambda^{3} u_{0}^{(1)}+m \lambda^{4}\right) \psi^{(1)}-G r t_{y}^{(1)} \\
i\left(\lambda u_{0}^{(1)} t^{(1)}+\lambda T_{0 y}^{(1)} \psi^{(1)}\right)=\frac{k}{\operatorname{Pr}}\left(-\lambda^{2} t^{(1)}+t_{y y}^{(1)}\right)
\end{gathered}
$$

$$
\begin{gathered}
\psi_{y y y y}^{(2)}-i\left(\lambda u_{0}^{(2)}+2 \lambda^{2}\right) \psi_{y y}^{(2)}+\left(i \lambda u_{0 y y}^{(2)}+i \lambda^{3} u_{0}^{(2)}+\lambda^{4}\right) \psi^{(2)}-G r h^{3} t_{y}^{(2)} \\
i\left(\lambda u_{0}^{(2)} t^{(2)}+\lambda T_{0 y}^{(2)} \psi^{(2)}\right)=\frac{1}{\operatorname{Pr}}\left(-\lambda^{2} t^{(2)}+t_{y y}^{(2)}\right)
\end{gathered}
$$

Boundary and interface conditions as defined in Eqs. (48) and (49) can be written in terms of $\psi^{(j)}$ and $t^{(j)}$ as

$$
\begin{gathered}
\frac{\partial \psi^{(1)}}{\partial y}=\cos (\theta) \frac{d u_{0}^{(1)}}{d y}, \psi^{(1)}=0 \quad \text { at } y=-1 ; \quad \frac{\partial \psi^{(2)}}{\partial y}=\frac{1}{h} \frac{d u_{0}^{(2)}}{d y}, \psi^{(2)}=0, \quad \text { at } y=1 \\
\psi_{y}^{(1)}=\frac{\psi_{y}^{(2)}}{h}, \psi^{(1)}=\frac{\psi^{(2)}}{h}, \psi_{y y}^{(1)}+\lambda^{2} \psi^{(1)}=\frac{\psi_{y y}^{(2)}+\lambda^{2} \psi^{(2)}}{m h^{2}} \text { at } y=0 \\
i \lambda \psi^{(1)} u_{0 y}^{(1)}-i \lambda u_{0}^{(1)} \psi_{y}^{(1)}+m\left(\psi_{y y y}^{(1)}-\lambda^{2} \psi_{y}^{(1)}\right)-\sigma^{2} \psi_{y}^{(1)}-G r t^{(1)}=\frac{1}{h^{3}}\left(i \lambda \psi^{(2)} u_{0 y}^{(2)}-i \lambda u_{0}^{(2)} \psi_{y}^{(2)}-\lambda^{2} \psi_{y}^{(2)}+\psi_{y y y}^{(2)}-G r h^{3} t^{(2)}\right) \text { at } y=0 \\
t^{(1)}=-\cos (\theta) \frac{d T_{0}^{(1)}}{d y} \text { at } y=-1 ; t^{(2)}=-\frac{1}{h} \frac{d T_{0}^{(2)}}{d y} \text { at } y=1 ; \quad t^{(1)}=t^{(2)}, \quad t_{y}^{(1)}+i \lambda t^{(1)}=\frac{t_{y}^{(2)}+i \lambda t^{(2)}}{k h} \text { at } y=0
\end{gathered}
$$

We restrict our attention to the real parts of the solutions for the perturbed quantities $\psi, t, u_{1}, v_{1}$ and $T_{1}$.

Consider only small values of $\lambda$ and on substituting

$$
\psi(\lambda, y)=\sum_{z=0}^{\infty} \lambda^{z} \psi_{z}, t(\lambda, y)=\sum_{z=0}^{\infty} \lambda^{z} t_{z}
$$

into Eqs. (52) to (57) we obtain to the order of $\lambda$, the following set of ordinary differential equations. Zeroth order

$$
\begin{gathered}
\frac{d^{2} t_{10}}{d y^{2}}=0 \\
m \frac{d^{4} \psi_{10}}{d y^{4}}-\sigma^{2} \frac{d^{2} \psi_{10}}{d y^{2}}-G r \frac{d t_{10}}{d y}=0
\end{gathered}
$$




$$
\begin{gathered}
\frac{d^{2} t_{20}}{d y^{2}}=0 \\
\frac{d^{4} \psi_{20}}{d y^{4}}-G r h^{3} \frac{d t_{20}}{d y}=0
\end{gathered}
$$

First order

$$
\begin{gathered}
\frac{d^{2} t_{11}}{d y^{2}}=i \frac{k}{\operatorname{Pr}}\left(u_{0}^{(1)} t_{10}+\frac{d T_{0}^{(1)}}{d y} \psi_{10}\right) \\
m \frac{d^{4} \psi_{11}}{d y^{4}}-\sigma^{2} \frac{d^{2} \psi_{11}}{d y^{2}}=i\left(u_{0}^{(1)} \frac{d^{2} \psi_{10}}{d y^{2}}-\frac{d^{2} u_{0}^{(1)}}{d y^{2}} \psi_{10}\right)+G r \frac{d t_{11}}{d y} \\
\frac{d^{2} t_{21}}{d y^{2}}=i \frac{1}{\operatorname{Pr}}\left(u_{0}^{(2)} t_{20}+\frac{d T_{0}^{(2)}}{d y} \psi_{20}\right) \\
\frac{d^{4} \psi_{21}}{d y^{4}}=i\left(u_{0}^{(2)} \frac{d^{2} \psi_{20}}{d y^{2}}-\frac{d^{2} u_{0}^{(2)}}{d y^{2}} \psi_{20}\right)+G r h^{3} \frac{d t_{21}}{d y}
\end{gathered}
$$

Zeroth order boundary and interface conditions in terms of stream function and temperature are

$$
\begin{gathered}
\frac{d \psi_{10}}{d y}=\cos (\theta) \frac{d u_{0}^{(1)}}{d y}, \psi_{10}=0 \text { at } y=-1 ; \quad \frac{d \psi_{20}}{d y}=\frac{1}{h} \frac{d u_{0}^{(2)}}{d y}, \psi_{20}=0 \text { at } y=1 \\
\frac{d \psi_{10}}{d y}=\frac{1}{h} \frac{d \psi_{20}}{d y}, \psi_{10}=\frac{1}{h} \psi_{20}, \frac{d^{2} \psi_{10}}{d y^{2}}=\frac{1}{m h^{2}} \frac{d^{2} \psi_{20}}{d y^{2}} \quad \text { at } y=0 \\
m \frac{d^{3} \psi_{10}}{d y^{3}}-\sigma^{2} \frac{d \psi_{10}}{d y}-G r t_{10}=\frac{1}{h^{3}}\left(\frac{d^{3} \psi_{20}}{d y^{3}}-G r h^{3} t_{20}\right) \text { at } \quad y=0 \\
t_{10}=-\cos (\theta) \frac{d T_{0}^{(1)}}{d y} \text { at } y=-1 ; \quad t_{20}=-\frac{1}{h} \frac{d T_{0}^{(2)}}{d y} \text { at } y=1 ; \quad t_{10}=t_{20}, \frac{d t_{10}}{d y}=\frac{1}{k h} \frac{d t_{20}}{d y} \text { at } y=0
\end{gathered}
$$

First order boundary and interface conditions in terms of stream function and temperature are

$$
\begin{gathered}
\frac{d \psi_{11}}{d y}=0, \psi_{11}=0 \text { at } y=-1 ; \frac{d \psi_{21}}{d y}=0, \psi_{21}=0 \text { at } y=1 \\
\frac{d \psi_{11}}{d y}=\frac{1}{h} \frac{d \psi_{21}}{d y}, \psi_{11}=\frac{\psi_{21}}{h}, \frac{d^{2} \psi_{11}}{d y^{2}}=\frac{1}{m h^{2}} \frac{d^{2} \psi_{21}}{d y^{2}} \text { at } y=0 \\
\frac{d u_{0}^{(1)}}{d y} \psi_{10}-i u_{0}^{(1)} \frac{d \psi_{10}}{d y}+m \frac{d^{3} \psi_{11}}{d y^{3}}-\sigma^{2} \frac{d \psi_{11}}{d y}-G r t_{11}=\frac{1}{h^{3}}\left(i \frac{d u_{0}^{(2)}}{d y} \psi_{20}-i u_{0}^{(2)} \frac{d \psi_{20}}{d y}+\frac{d^{3} \psi_{21}}{d y^{3}}-G r h^{3} t_{21}\right) \text { at } y=0 \\
t_{11}=0 \text { at } y=-1 ; t_{21}=0 \text { at } y=1 ; \quad t_{11}=t_{21}, \frac{d t_{11}}{d y}+i t_{10}=\frac{1}{k h}\left(\frac{d t_{21}}{d y}+i t_{20}\right) \text { at } y=0
\end{gathered}
$$

The set of Eqs. (29) to (32) subjected to boundary and interface conditions (39) and (40) have been solved exactly for $u_{0}^{(j)}$ and $T_{0}^{(j)}$, and the set of Eqs. (59) and (60) subject to boundary and interface conditions (61) and (62) have been solved for $\psi_{j}$ and $t_{j}$ $(j=1,2)$. From these solutions, the first order quantities can be put in the form,

$$
\psi_{j}=\left(\psi_{r}+i \psi_{i}\right)_{j}=\psi_{j 0}+\lambda \psi_{j 1}, \quad t_{j}=\left(t_{r}+i t_{i}\right)_{j}=t_{j 0}+\lambda t_{j 1}(j=1,2)
$$

where suffix $r$ denotes the real part and $i$ denotes the imaginary part

Considering only real part, the expression for first order velocity and temperature become

$$
\begin{gathered}
u_{1}^{(j)}=\varepsilon\left(\lambda \sin (\lambda x) \frac{d \psi_{i}^{(j)}}{d y}-\cos (\lambda x) \frac{d \psi_{r}^{(j)}}{d y}\right) \\
v_{1}^{(j)}=\varepsilon\left(-\lambda \psi_{r}^{(j)} \sin (\lambda x)-\lambda^{2} \psi_{i}^{(j)} \cos (\lambda x)\right) \\
T_{1}^{(j)}=\varepsilon\left(\cos (\lambda x) t_{r}^{(j)}-\lambda \sin (\lambda x) t_{i}^{(j)}\right)
\end{gathered}
$$

The total solutions for the velocity and temperature become the summation of the mean and perturbed part.

The solutions and constants are given in the appendix section. 


\subsection{Skin friction and Nusselt number.}

The shearing stress $\tau_{x y}$ at any point in the fluid is given in non-dimensional form, by

$$
\tau_{x y}=\left(\frac{h^{2}}{\rho v^{2}}\right) \bar{\tau}_{x y}=\frac{\partial u}{\partial y}+\frac{\partial v}{\partial x}
$$

At the wavy walls, $y=-1+\varepsilon \cos (\lambda x+\theta)$ and $y=1+\frac{\varepsilon \cos (\lambda x)}{h}$, the skin friction $\tau_{x y}$ becomes

$$
\tau_{-1}=\tau_{-1}^{0}+\varepsilon\left(\cos (\lambda x+\theta) u_{0}^{\prime \prime}(-1)+u_{1}^{\prime}(-1)\right)
$$

and

$$
\tau_{1}=\tau_{1}^{0}+\varepsilon\left(\frac{1}{h} \cos (\lambda x) u_{0}^{\prime \prime}(1)+u_{1}^{\prime}(1)\right)
$$

respectively, where

$$
\tau_{-1}^{0}=\frac{d u_{0}^{(1)}}{d y}(-1) \text { and } \tau_{1}^{0}=\frac{d u_{0}^{(2)}}{d y}(1)
$$

The dimensionless Nusselt number is given by

$$
N u=\frac{\partial T}{\partial y}=T_{0}^{\prime}(y)+\varepsilon \operatorname{Re}\left(e^{i \lambda x} T_{1}^{\prime}(y)\right)
$$

At the wavy walls, $y=-1+\varepsilon \cos (\lambda x+\theta)$ and $y=1+\frac{\varepsilon \cos (\lambda x)}{h}$, Eqn. (70) assumes the form

$$
N u_{-1}=N u_{-1}^{0}+\varepsilon\left(\cos (\lambda x+\theta) T_{0}^{(1) "}(-1)+t^{\prime}(-1)\right)
$$

and

$$
N u_{1}=N u_{1}^{0}+\varepsilon\left(\frac{1}{h} \cos (\lambda x) T_{0}^{(2)^{\prime}}(1)+t_{1}^{\prime}(1)\right)
$$

respectively, where

$$
N u_{-1}^{0}=\frac{d T_{0}^{(1)}}{d y}(-1) \text { and } N u_{1}^{0}=\frac{d T_{0}^{(2)}}{d y}(1)
$$

where Re represents the real part

The expressions for $\tau_{-1}^{0}, \tau_{1}^{0}, N u_{-1}^{0}$ and $N u_{1}^{0}$ are obtained from zeroth order solutions $u_{0}$ and $T_{0}$ and are numerically evaluated for several sets of values of the parameters $m, h, k$ and $\theta$. Also, the wall skin friction $\tau_{-1}, \tau_{1}$ and the wall Nusselt number $N u_{-1}$ and $N u_{1}$ are calculated numerically and some of the qualitative interesting features are presented graphically.

\section{Results and discussion}

Mixed convective flow and heat transfer of composite porous medium in a vertical wavy channel is studied analytically. The parameters such as Prandtl number, wave number, amplitude parameter and $\lambda x$ are fixed as $0.7,0.05,0.02,0.785398$ respectively for all the computations, whereas Grashof number, viscosity ratio, width ratio, conductivity ratio and traveling thermal temperature are fixed as $5,1,1,1,0.785398$ respectively for all the graphs except the varying one. The effect of porous parameter $\sigma$ is observed for all the graphs from Figures 2 to 11 and Tables 1 to 3.

The effect of increasing Grashof number $G r$ is to increase the fluid motion for zeroth order velocity $u_{0}$ as seen in Figure 2a. It is also observed that for large porous parameter $\sigma$, frictional drag resistance against the flow motion becomes pronounced and as a result, the velocity generally reduced in porous region. As the porous parameter $\sigma$ increases velocity decreases significantly in the permeable fluid layer. With the dragging effect across the interface the velocity in the region-II also decreases as $\sigma$ increases. The first order velocity decreases in region-I ( $y=-1$ to 0 approximately) and increases in region-II with the Grashof number increases as seen in Figure 2b. The first order velocity $u_{1}$ is pronounced as the porous parameter $\sigma$ increases, in the region-I ( $y=-1$ to 0 approximately) whereas, as $\sigma$ increases, first order velocity decreases in the region-II. The velocity $u$ for different values of Grashof number and porous parameter is shown in Figure 2c. The effect of $G r$ and $\sigma$ on velocity $u$ parallel to the flow direction is similar to the effect on zeroth order velocity $u_{0}$. Physically, an increase in the value of the Grashof number means an increase in the buoyancy force which supports the motion. The behavior of the fluid velocity $v$ perpendicular to the channel length on the Grashof number and porous parameter is observed in Figure 2d. As the Grashof number increases velocity $v$ decreases whereas as the porous parameter $\sigma$ increases, velocity increases. 
The effect of viscosity ratio $m\left(=\mu_{\text {eff }} / \mu\right)$ on the velocity $u$ and $v$ is shown in Figure 3 . As the viscosity ratio $m$ increases zeroth order velocity decreases in both the regions as seen in Figure 3a. It is also observed that as the porous parameter $\sigma$ increases, zeroth order velocity decreases in both the regions. However, the effect of $\sigma$ is more operative in region-I when compared to region-II. The effect of viscosity ratio on first order velocity $u_{1}$ is to enhance the velocity in the porous region in magnitude and suppress the velocity in the viscous region as seen in Figure 3b. The effect of viscosity ratio $m$ and porous parameter $\sigma$ on the velocity $u$ is again similar to the effect on zeroth order velocity $u_{0}$ as seen in Figure 3c. Physically, the increase in viscosity ratio means, fluid become thicker which will reduce the flow filed. The fluid velocity $v$ perpendicular to the channel length increases as the viscosity ratio $m$ and porous parameter $\sigma$ increases as seen in Figure 3d.
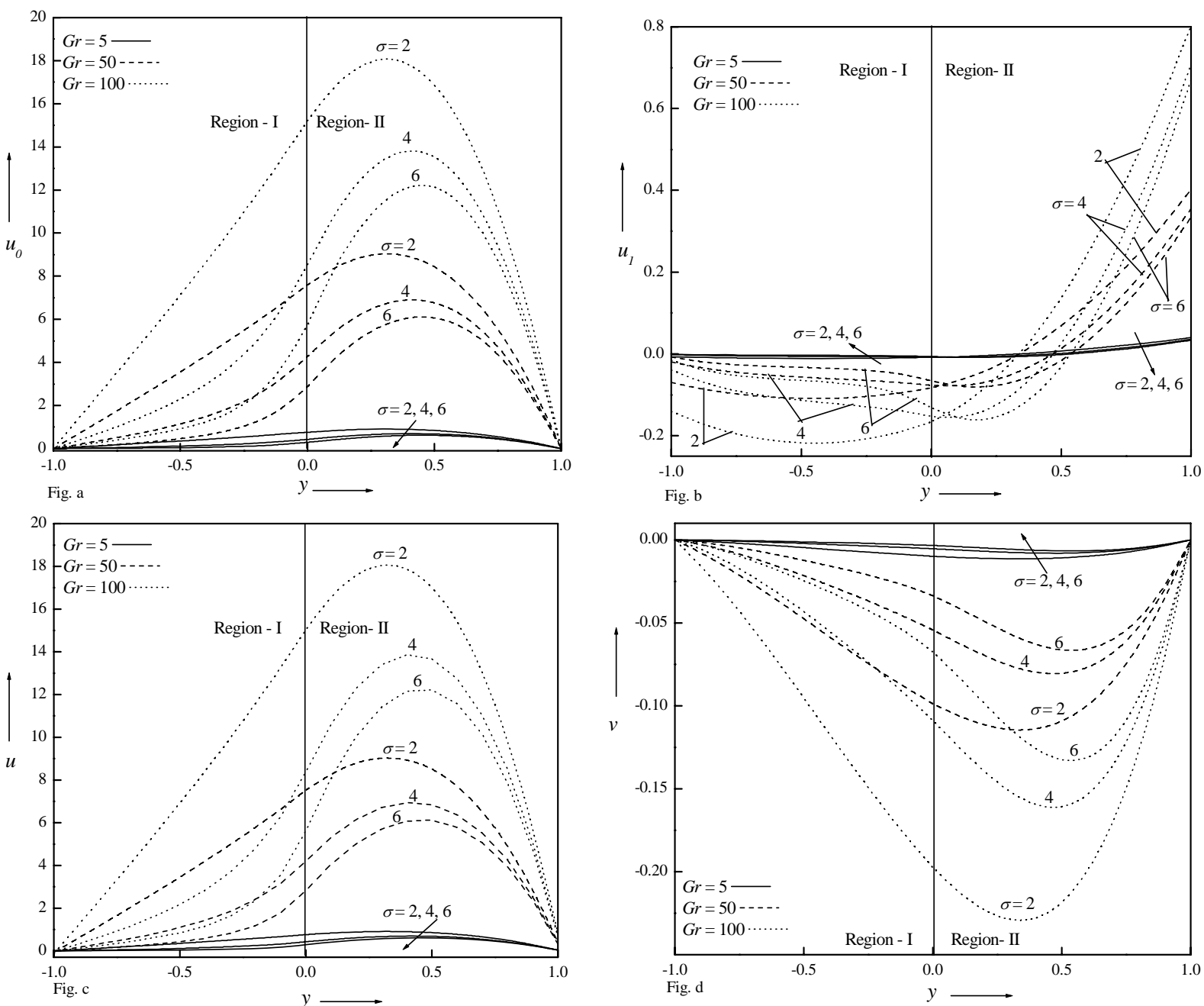

Figure 2: Velocity profiles for different values of Grashof number and porous parameter.

(a) zeroth order profiles, (b) first order profiles, (c) velocity profiles in $u$ and (d) velocity profiles in $v$.

The influence of width ratio $h\left(=h^{(2)} / h^{(1)}\right)$ on the velocity field is displayed in Figure 4 . The effect of width ratio $h$ on zeroth order velocity $u_{0}$ is to enhance the velocity in both the regions. That is larger the width of the clear viscous fluid layer compared to width of the permeable fluid layer, the stronger the flow field. It is observed that the width ratio $h$ is more effective in viscous fluid region compared to permeable fluid region. The effect of porous parameter $\sigma$ on zeroth order velocity is to reduce the velocity in both the regions as seen in Figure 4a. The effect of width ratio $h$ on the first order velocity $u_{1}$ is not significant in region-I compared to region-II whereas, as the width ratio increases, velocity increases in region-II. The effect of porous parameter $\sigma$ is to decrease the first order velocity $u_{1}$ in region-II and its effect is not significant in region-I as seen in Figure $4 \mathrm{~b}$. The effect of width ratio $h$ and porous parameter $\sigma$ on the velocity $u$ is exactly similar to the effect on zeroth order velocity $u_{0}$ as observed 
in Figure 4c. The effect of width ratio $h$ and porous parameter $\sigma$ on fluid velocity $v$ is shown in Figure 4d. As the width ratio increases velocity $v$ decreases for small porous parameter and it increases for porous parameter $\sigma \geq 4$ in porous region, in viscous region, the width ratio reduces the velocity $v$ whereas, the porous parameter $\sigma$ enhance the fluid velocity $v$.
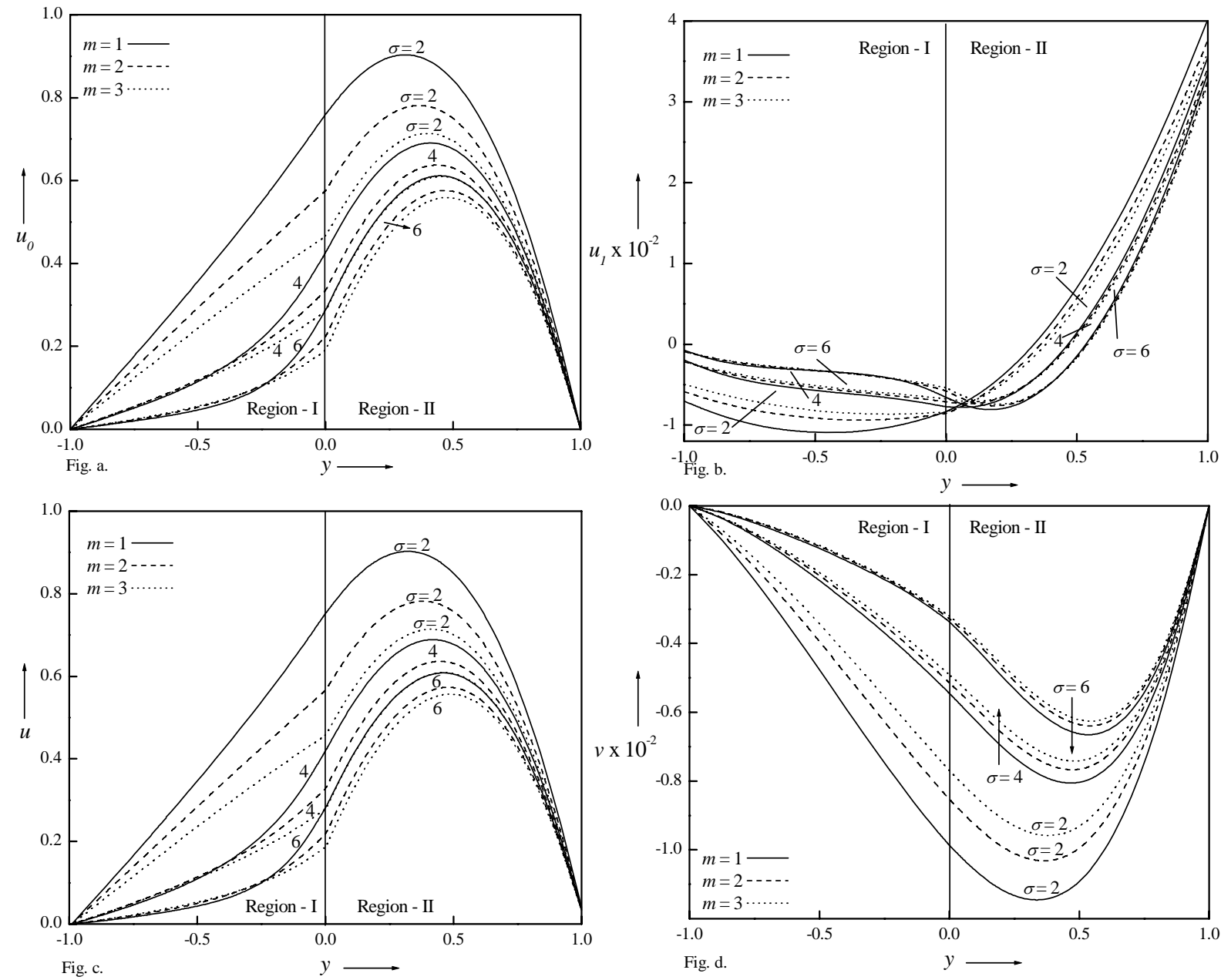

Figure 3: Velocity profiles for different values of viscosity ratio and porous parameter.

(a) zeroth order profiles, (b) first order profiles, (c) velocity profiles in $u$ and (d) velocity profiles in $v$.

The influence of width ration $h$ on the temperature field is to decrease the zeroth order temperature field $T_{0}$, as displayed in Figure 5a. Figure $5 \mathrm{~b}$ reflects that as the width ratio $h$ increases the first order temperature $T_{1}$ increases. The effect of width ratio $h$ on temperature $T$ is similar to its effect on zeroth order temperature $T_{0}$. It is also observed from Figure $5 \mathrm{a}$ and $5 \mathrm{c}$ that the effect of porous parameter $\sigma$ does not affect the temperature field whereas, first order temperature decreases to the order of $10^{-3}$ as porous parameter increases as seen in Figure $5 b$.

The role of conductivity ratio $k\left(=K_{\text {eff }} / K\right)$ and porous parameter $\sigma$ is to suppress the zeroth order velocity $u_{0}$ as seen in Figure 6a. The first order velocity $u_{1}$ increases in region-I and decreases in region-II as the conductivity ratio $k$ and porous parameter $\sigma$ increases as seen in Figure 6b. The effect of $k$ and $\sigma$ on the velocity $u$ is similar to the effect on the zeroth order velocity. Physically, larger the conductivity of the porous matrix compared to fluid, the smaller the flow field. The fluid velocity $v$ enhances as the conductivity ratio $k$ and porous parameter increases as seen in Figure 6d.

The effect of conductivity ratio $k$ on zeroth order and total temperature $T$ is similar to the effect of width ratio $h$ (Figure 5) as seen in Figures 7a and 7c. That is, as the conductivity ratio increases, zeroth and total temperature decreases. The effect of $k$ on first order temperature $T_{1}$ decreases as conductivity ratio increases as seen in Figure $7 \mathrm{~b}$. It is also observed from Figure 7a and 7c 
that the effect of porous parameter $\sigma$ does not affect the temperature field whereas, as the porous parameter $\sigma$ increases first order temperature decreases as seen in Figure $7 \mathrm{~b}$.
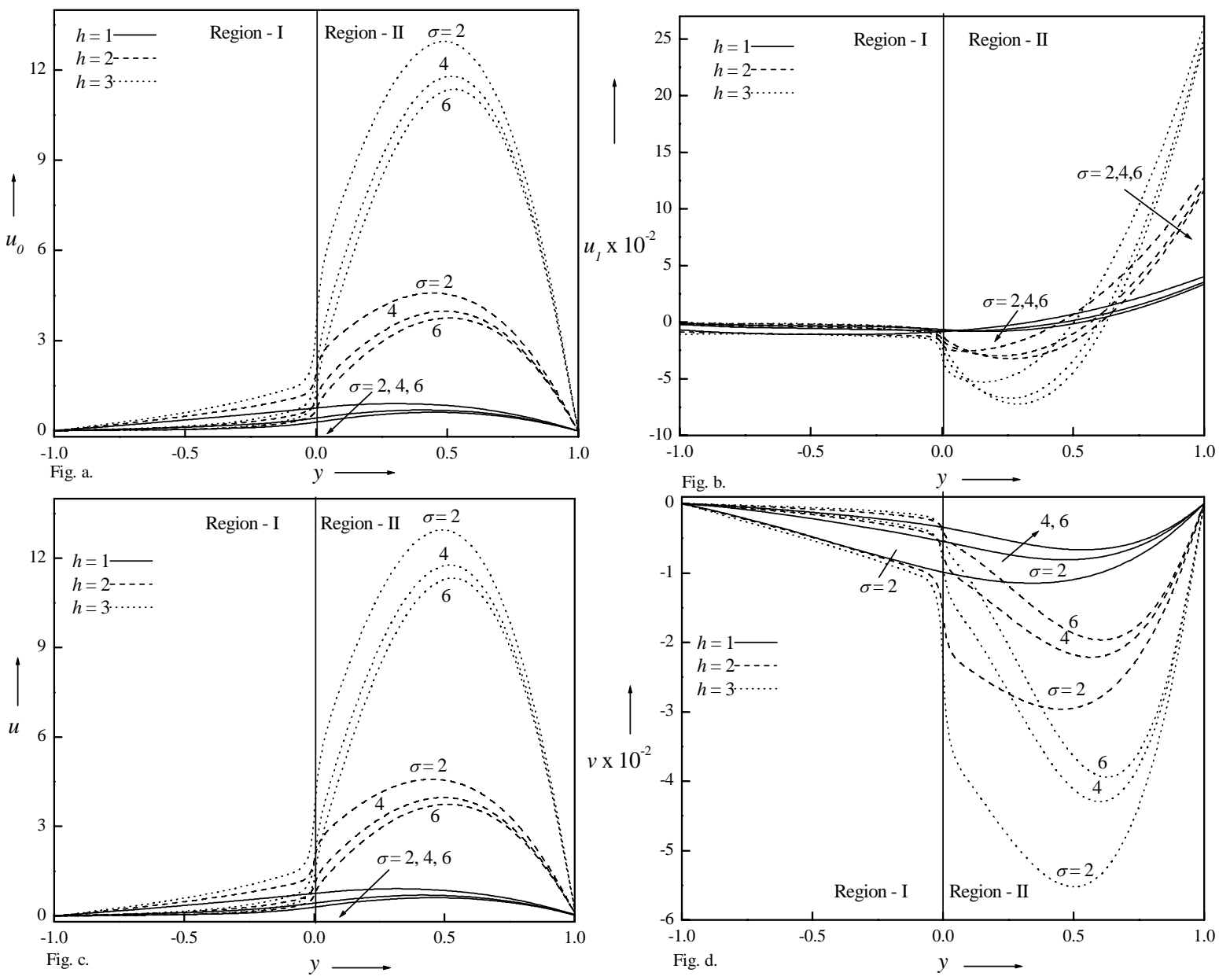

Figure 4: Velocity profiles for different values of width ratio and porous parameter.

(a) zeroth order profiles, (b) first order profiles, (c) velocity profiles in $u$ and (d) velocity profiles in $v$.

The effect of the traveling thermal temperature $\theta$ on first order velocity $u_{1}$, and velocity $u$ is shown in Figure 8 . The first order velocity $u_{1}$ increases as traveling thermal temperature $\theta$ increases from $y=-1$ to -0.25 approximately and decreases from $y=-0.25$ onwards as seen in Figure 8a. The velocity $u$ increases as the traveling thermal temperature $\theta$ increases in region-I near the left wavy wall and decreases in region-II near the interface as seen in Figure 8b. The fluid velocity $v$ perpendicular to the channel increases as the traveling thermal temperature $\theta$ increases as seen in Figure 8c. Its effect is more significant near the left wavy wall.

The effect of traveling thermal temperature $\theta$ on first order and total temperature is shown in Figure 9. First order temperature increases as the traveling thermal temperature $\theta$ increases and its effect is more in region-I compared to region-II as seen in Figure 9a. The effect of the traveling thermal temperature $\theta$ on total temperature increases slightly near the left wavy wall and remains constant at the right wavy wall as seen in Figure $9 \mathrm{~b}$. 

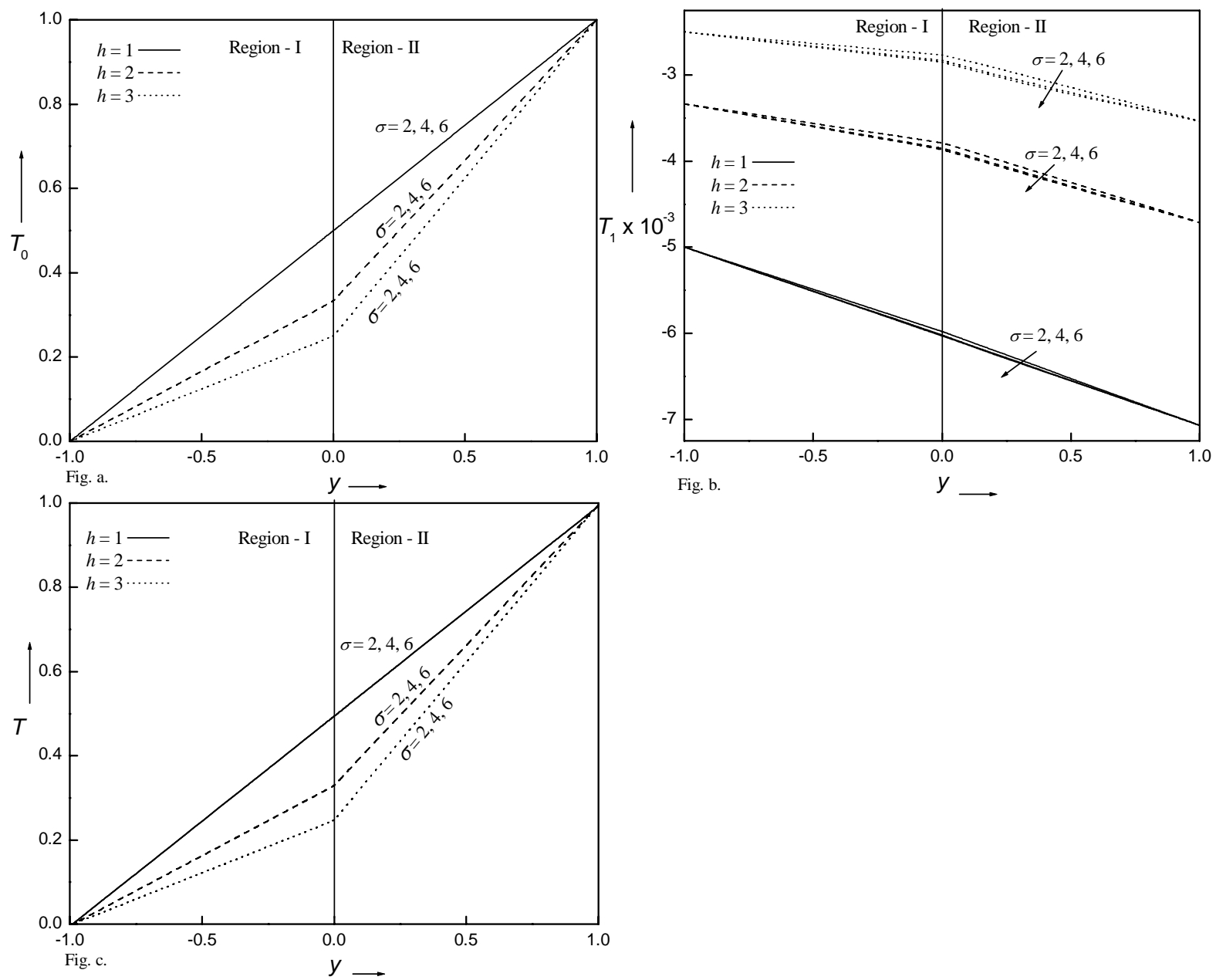

Figure 5: Temperature profiles for different values of width ratio and porous parameter. (a) zeroth order, (b) first order and (c) total temperature.

Shear stress $\tau$ at the walls of the channel is analysed for different values of Grashof number $G r$, width ratio $h$ and porous parameter $\sigma$ and is shown in Figure 10. As the Grashof number $G r$ and width ratio $h$ increases the skin friction at the left wall increases and decrease at the right wall. The porous parameter decreases the skin friction at the left wall and increases in magnitude at the right wall as seen in Figure 10.

The heat transfer coefficient $\mathrm{Nu}$ for different values of $\mathrm{Gr}$ and width ratio $h$ is shown in Figure 11. The Nusselt number at the left wavy wall $\mathrm{Nu}_{-1}$ and Nusselt number at the right wavy wall $\mathrm{Nu}_{1}$ remains invariant on Grashof number. Varying the width ratio $h$, the Nusselt number at the left wavy wall is vary large compare to the Nusselt number at the right wavy wall.

Table 1 depicts the effect of amplitude and porous parameter on Nusselt number distribution for fixed values of $G r=10$, $m=h=k=1, \operatorname{Pr}=0.7, \lambda x=\pi / 4$ and $\theta=0$. It is noted that the $N u_{-1}$ decreases and $N u_{1}$ increases with increase in amplitude (or wave number) and porous parameter, which is the similar result obtained by Jang et al. (2003, 2004). (That is the Nusselt number is small for large amplitude-wavelength ratio). Also, the Nusselt number decreases as the wavelength increases as observed by Varol and Oztop, (2006). 

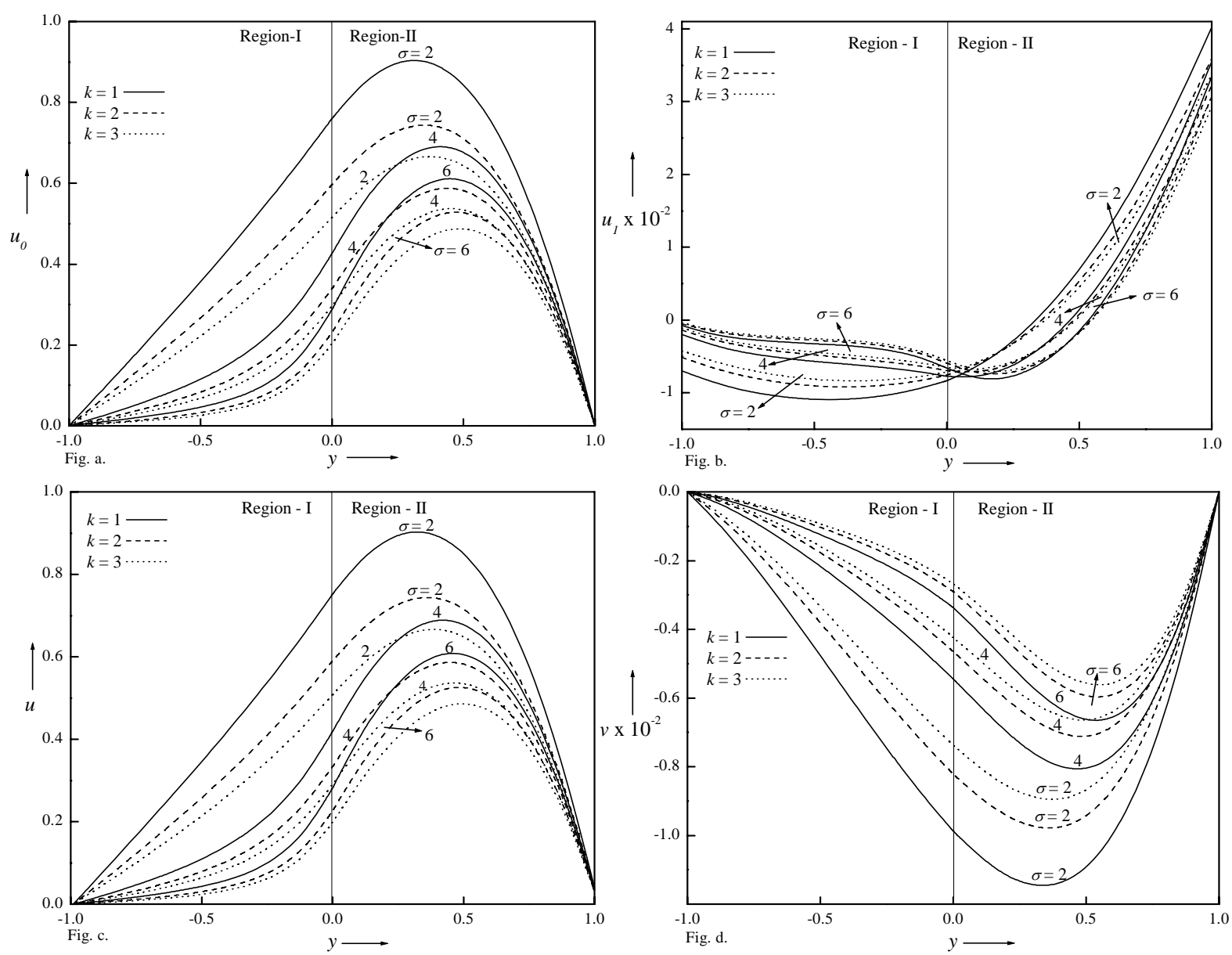

Figure 6: Velocity profiles for different values of conductivity ratio and porous parameter.

(a) zeroth order profiles, (b) first order profiles, (c) velocity profiles in $u$ and (d) velocity profiles in $v$.

The effect of convective parameter $\mathrm{Gr}$ on temperature is shown in Table 2. The zeroth order temperature equation does not contain Grashof number and hence remains invariant for the effects of Grashof number. However Grashof number occurs in the first order temperature equation through zeroth order velocity. It is observed that the first order temperature increases to the order of $10^{-5}$ with increase in the Grashof number. The effect of Grashof number on total temperature also increases to the order of $10^{-5}$. The porous parameter $\sigma$ decreases the first order temperature and total temperature as seen in Table 2 .

The effect of viscosity ratio on the temperature field is shown in Table 3. It is observed that zeroth order temperature is invariant on viscosity ratio whereas, first order temperature varies to the order of $10^{-3}$. However the effect of viscosity ratio on total temperature also varies to the order of $10^{-3}$ and there is no effect of porous parameter $\sigma$ on zeroth, first order and total temperature as seen in Table 3.

To validate the results for the present model, the results are compared with Umavathi and Shekar (2011) and (Vajravelu and Sastri, 1978). Considering purely viscous fluid in region-I, the present model will reduce to Umavathi and Shekar (2011). For values of viscosity ratio, width ratio and conductivity ratio to be one and in the absence of porous parameter with $\lambda x=\pi / 2$ and $\theta=0$ will reduce the present model to one-fluid model (Vajravelu and Sastri, 1978). The results of velocity $u$ and temperature $T$ agree very well with Umavathi and Shekar (2011) and Vajravelu and Sastri (1978) as seen in Table 4. For comparison of the present model with Vajravelu and Sastri (1978), the problem of Vajravelu and Sastri (1978) is solved in the absence of heat source/sink, the plates are placed at $y=-1+\varepsilon \cos (\lambda x)$ instead of $y=\varepsilon \cos (\lambda x)$ and boundary conditions on temperature are chosen to be $T=0$ at $y=-1+\varepsilon \cos (\lambda x)$ and $T=1$ at $y=1$. 

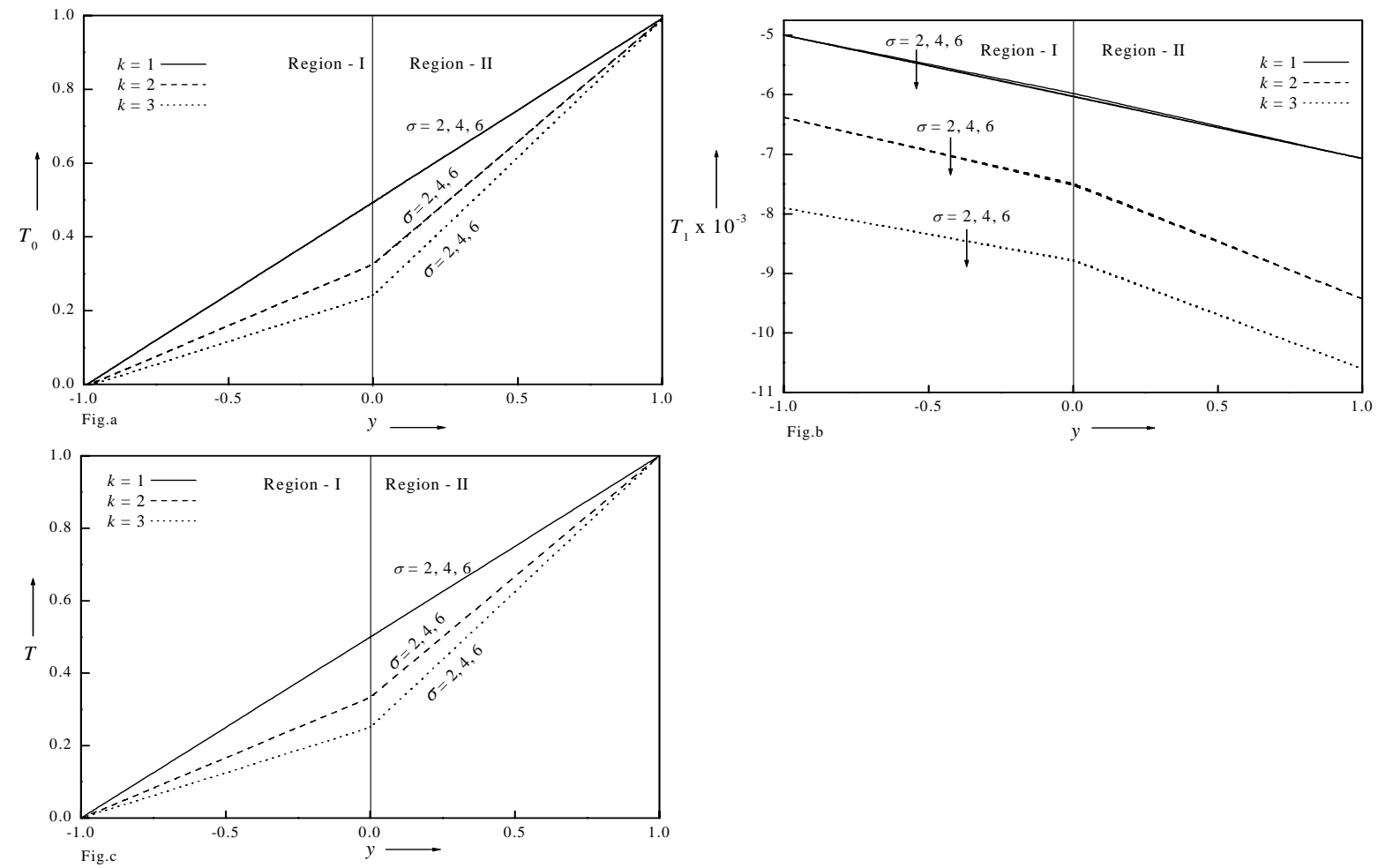

Figure 7: Temperature profiles for different values of conductivity ratio and porous parameter. (a) zeroth order, (b) first order and (c) total temperature profiles.
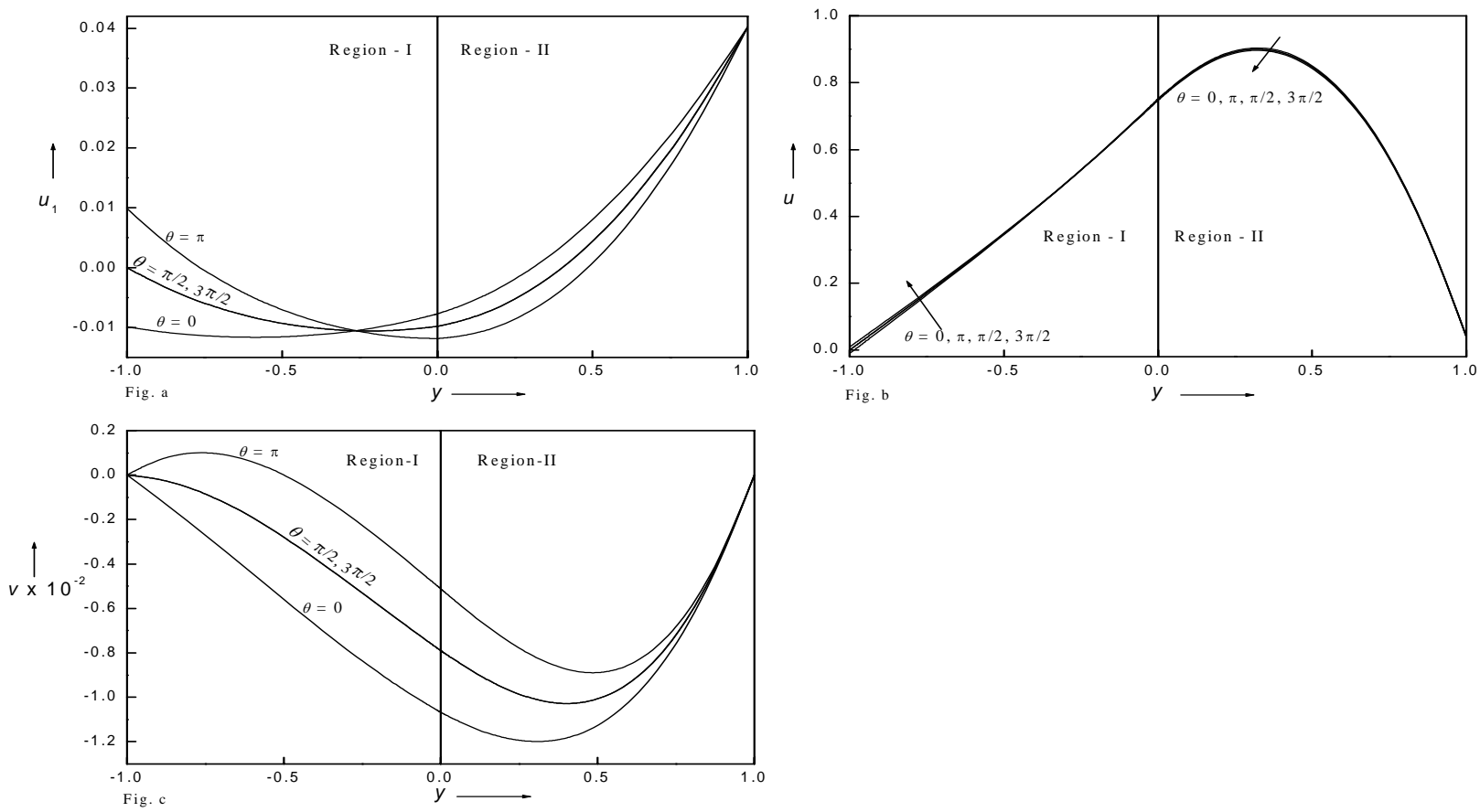

Figure 8: Velocity profiles for different values of traveling thermal temperature $\theta$. (a) velocity in $u$, (b) first order velocity and (c) velocity in $v$. 



Figure 9: Temperature profiles for different values of traveling thermal temeprature $\theta$.

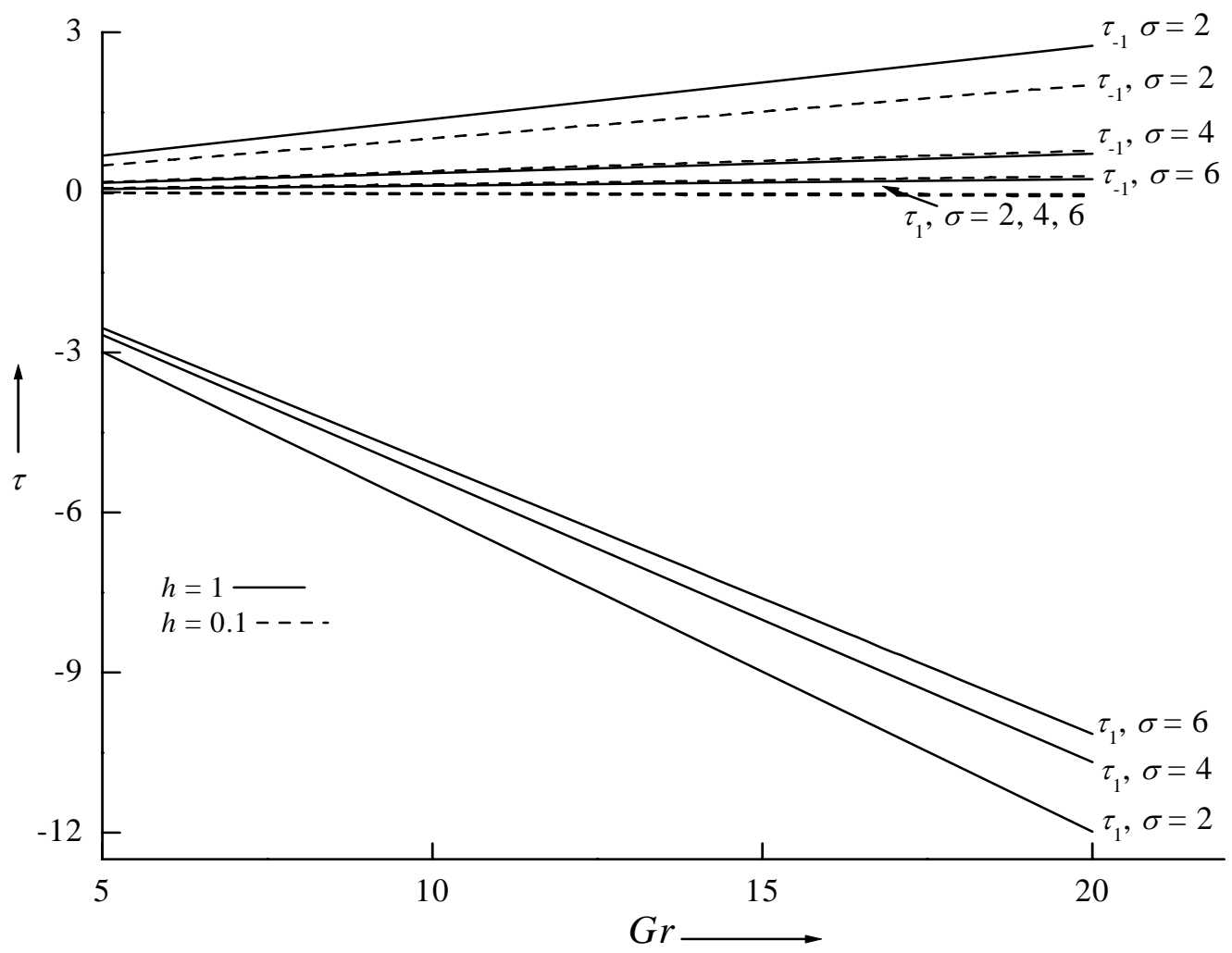

Figure 10: skin friction profiles for different values of width ratio and porous parameter. 


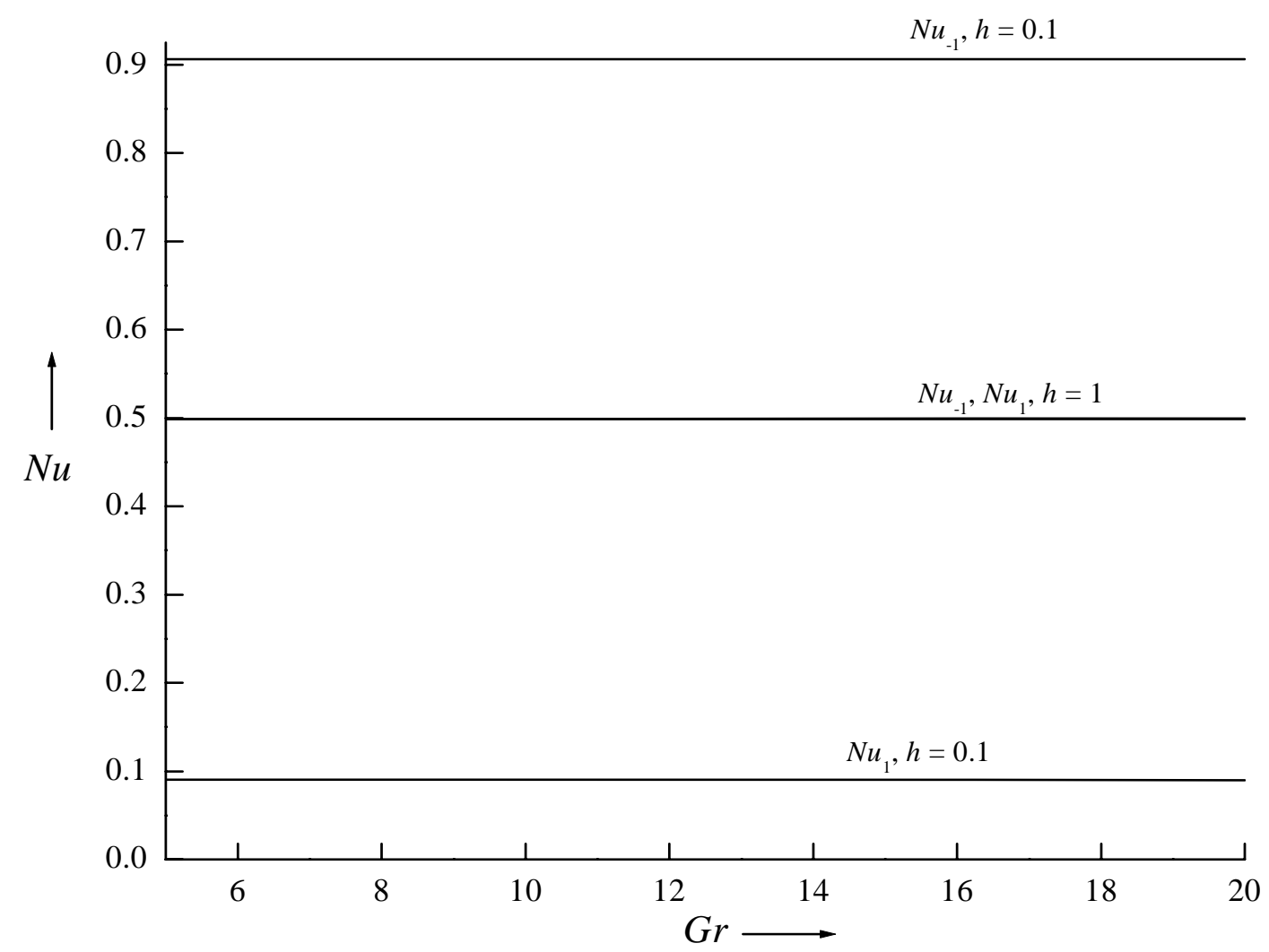

Figure 11: Nusselt number profiles for different values of width ratio.

Table 1. Values of the Nusselt number for different values of amplitude, $\lambda x$ and porous parameter with $m=k=h=1$.

\begin{tabular}{|c|c|c|c|c|c|c|c|c|}
\hline & \multicolumn{4}{|c|}{$\varepsilon=0.02, \lambda=0.05$} & \multicolumn{4}{c|}{$\varepsilon=1.0, \lambda=1.0$} \\
\cline { 2 - 9 } & \multicolumn{2}{|c|}{ Flat walls } \\
& $(\lambda x=\pi / 2, \theta=0)$ & \multicolumn{2}{c|}{$\begin{array}{c}\text { Wavy walls } \\
(\lambda x=\pi / 4, \theta=0)\end{array}$} & \multicolumn{2}{c|}{$\begin{array}{c}\text { Flat walls } \\
(\lambda x=\pi / 2, \theta=0)\end{array}$} & \multicolumn{2}{c|}{$\begin{array}{c}\text { Wavy walls } \\
(\lambda x=\pi / 4, \theta=0)\end{array}$} \\
\hline$\sigma$ & $N u_{-1}$ & $N u_{1}$ & $N u_{-1}$ & $N u_{1}$ & $N u_{-1}$ & $N u_{1}$ & $N u_{-1}$ & $N u_{1}$ \\
\hline 10.0 & 0.499991 & 0.500047 & 0.499994 & 0.500033 & 0.491215 & 0.547170 & 0.493788 & 0.533354 \\
\hline 20.0 & 0.499976 & 0.500064 & 0.499983 & 0.500045 & 0.475529 & 0.564109 & 0.482696 & 0.545332 \\
\hline 30.0 & 0.499973 & 0.500066 & 0.499981 & 0.500047 & 0.472535 & 0.566010 & 0.480579 & 0.546676 \\
\hline
\end{tabular}

Table 2. Values of the Temperature field for different values of Grashof number and porous parameter.

\begin{tabular}{|l|l|l|l|l|c|c|c|}
\hline \multicolumn{7}{|c|}{$y$} & \multicolumn{2}{|c|}{$T_{0}$} & \multicolumn{3}{c|}{$T_{1}$} & \multicolumn{3}{c|}{$T$} \\
\cline { 2 - 8 } & $G r=5,50,100$ & $G r=5$ & $G r=50$ & $G r=100$ & $G r=5$ & $G r=50$ & $G r=100$ \\
\hline-1 & 0 & -0.005 & -0.005 & -0.005 & -0.0050 & -0.0050 & -0.0050 \\
\hline-0.75 & 0.125 & -0.00526 & -0.00524 & -0.00523 & 0.11974 & 0.11976 & 0.11978 \\
\hline-0.5 & 0.25 & -0.00551 & -0.00548 & -0.00544 & 0.24449 & 0.24452 & 0.24456 \\
\hline-0.25 & 0.375 & -0.00577 & -0.00573 & -0.00568 & 0.36923 & 0.36927 & 0.36932 \\
\hline 0 & 0.5 & -0.00603 & -0.00599 & -0.00595 & 0.49397 & 0.49401 & 0.49405 \\
\hline 0.25 & 0.625 & -0.00629 & -0.00626 & -0.00623 & 0.61871 & 0.61874 & 0.61877 \\
\hline 0.5 & 0.75 & -0.00655 & -0.00653 & -0.00651 & 0.74345 & 0.74347 & 0.74349 \\
\hline 0.75 & 0.875 & -0.00681 & -0.00680 & -0.00679 & 0.86819 & 0.86820 & 0.86821 \\
\hline 1 & 1 & -0.00707 & -0.00707 & -0.00707 & 0.99293 & 0.99293 & 0.99293 \\
\hline
\end{tabular}




\begin{tabular}{|l|l|l|l|l|l|l|l|}
\hline \multicolumn{7}{|c|}{$\sigma=4$} \\
\hline-1 & 0 & -0.005 & -0.005 & -0.005 & -0.0050 & -0.0050 & -0.0050 \\
\hline-0.75 & 0.125 & -0.00526 & -0.00525 & -0.00523 & 0.11974 & 0.11975 & 0.11977 \\
\hline-0.5 & 0.25 & -0.00551 & -0.00549 & -0.00547 & 0.24448 & 0.24451 & 0.24453 \\
\hline-0.25 & 0.375 & -0.00577 & -0.00575 & -0.00573 & 0.36923 & 0.36925 & 0.36927 \\
\hline 0 & 0.5 & -0.00603 & -0.00602 & -0.00601 & 0.49397 & 0.49398 & 0.49399 \\
\hline 0.25 & 0.625 & -0.00629 & -0.00630 & -0.00630 & 0.61871 & 0.61870 & 0.61870 \\
\hline 0.5 & 0.75 & -0.00655 & -0.00656 & -0.00657 & 0.74345 & 0.74344 & 0.74343 \\
\hline 0.75 & 0.875 & -0.00681 & -0.00682 & -0.00682 & 0.86819 & 0.86818 & 0.86818 \\
\hline 1 & 1 & -0.00707 & -0.00707 & -0.00707 & 0.99293 & 0.99293 & 0.99293 \\
\hline \multicolumn{7}{|c|}{$\sigma=6$} \\
\hline-1 & 0 & -0.005 & -0.005 & -0.005 & -0.0050 & -0.0050 & -0.0050 \\
\hline-0.75 & 0.125 & -0.00526 & -0.00526 & -0.00526 & 0.11974 & 0.11974 & 0.11975 \\
\hline-0.5 & 0.25 & -0.00552 & -0.00551 & -0.00551 & 0.24448 & 0.24449 & 0.24449 \\
\hline-0.25 & 0.375 & -0.00577 & -0.00578 & -0.00578 & 0.36922 & 0.36922 & 0.36922 \\
\hline 0 & 0.5 & -0.00604 & -0.00606 & -0.00608 & 0.49396 & 0.49394 & 0.49392 \\
\hline 0.25 & 0.625 & -0.00630 & -0.00633 & -0.00636 & 0.61870 & 0.61867 & 0.61864 \\
\hline 0.5 & 0.75 & -0.00656 & -0.00659 & -0.00662 & 0.74344 & 0.74341 & 0.74338 \\
\hline 0.75 & 0.875 & -0.00681 & -0.00683 & -0.00685 & 0.86819 & 0.86817 & 0.86815 \\
\hline 1 & 1 & -0.00707 & -0.00707 & -0.00707 & 0.99293 & 0.99293 & 0.99293 \\
\hline
\end{tabular}

Table 3. Values of the Temperature field for different values of viscosity ratio and porous parameter.

\begin{tabular}{|c|c|c|c|c|c|c|c|}
\hline \multicolumn{8}{|c|}{$\sigma=2$} \\
\hline \multirow{2}{*}{$y$} & $T_{0}$ & \multicolumn{3}{|c|}{$T_{1}$} & \multicolumn{3}{|c|}{$T$} \\
\hline & $m=0.1,1,2$ & $m=0.1$ & $m=1$ & $m=2$ & $m=0.1$ & $m=1$ & $m=2$ \\
\hline-1 & 0 & -0.005 & -0.005 & -0.005 & -0.005 & -0.005 & -0.005 \\
\hline-0.75 & 0.125 & -0.00525 & -0.00526 & -0.00526 & 0.11975 & 0.11974 & 0.11974 \\
\hline-0.5 & 0.25 & -0.0055 & -0.00551 & -0.00551 & 0.2445 & 0.24449 & 0.24449 \\
\hline-0.25 & 0.375 & -0.00576 & -0.00577 & -0.00577 & 0.36924 & 0.36923 & 0.36923 \\
\hline 0 & 0.5 & -0.00602 & -0.00603 & -0.00603 & 0.49398 & 0.49397 & 0.49397 \\
\hline 0.25 & 0.625 & -0.00629 & -0.00629 & -0.00629 & 0.61871 & 0.61871 & 0.61871 \\
\hline 0.5 & 0.75 & -0.00655 & -0.00655 & -0.00655 & 0.74345 & 0.74345 & 0.74345 \\
\hline 0.75 & 0.875 & -0.00681 & -0.00681 & -0.00681 & 0.86819 & 0.86819 & 0.86819 \\
\hline 1 & 1 & -0.00707 & -0.00707 & -0.00707 & 0.99293 & 0.99293 & 0.99293 \\
\hline \multicolumn{8}{|c|}{$\sigma=4$} \\
\hline-1 & 0 & -0.005 & -0.005 & -0.005 & -0.005 & -0.005 & -0.005 \\
\hline-0.75 & 0.125 & -0.00526 & -0.00526 & -0.00526 & 0.11974 & 0.11974 & 0.11974 \\
\hline-0.5 & 0.25 & -0.00552 & -0.00552 & -0.00551 & 0.24448 & 0.24448 & 0.24449 \\
\hline-0.25 & 0.375 & -0.00578 & -0.00577 & -0.00577 & 0.36922 & 0.36923 & 0.36923 \\
\hline 0 & 0.5 & -0.00604 & -0.00603 & -0.00603 & 0.49396 & 0.49397 & 0.49397 \\
\hline 0.25 & 0.625 & -0.0063 & -0.00629 & -0.00629 & 0.6187 & 0.61871 & 0.61871 \\
\hline 0.5 & 0.75 & -0.00656 & -0.00655 & -0.00655 & 0.74344 & 0.74345 & 0.74345 \\
\hline 0.75 & 0.875 & -0.00682 & -0.00681 & -0.00681 & 0.86818 & 0.86819 & 0.86819 \\
\hline 1 & 1 & -0.00707 & -0.00707 & -0.00707 & 0.99293 & 0.99293 & 0.99293 \\
\hline \multicolumn{8}{|c|}{$\sigma=6$} \\
\hline-1 & 0 & -0.005 & -0.005 & -0.005 & -0.005 & -0.005 & -0.005 \\
\hline-0.75 & 0.125 & -0.00526 & -0.00526 & -0.00526 & 0.11974 & 0.11974 & 0.11974 \\
\hline-0.5 & 0.25 & -0.00552 & -0.00552 & -0.00552 & 0.24448 & 0.24448 & 0.24448 \\
\hline-0.25 & 0.375 & -0.00578 & -0.00578 & -0.00578 & 0.36922 & 0.36922 & 0.36922 \\
\hline 0 & 0.5 & -0.00605 & \begin{tabular}{|c|}
-0.00604 \\
\end{tabular} & -0.00604 & 0.49395 & 0.49396 & 0.49396 \\
\hline 0.25 & 0.625 & -0.00631 & -0.00631 & -0.00631 & 0.61869 & 0.6187 & 0.6187 \\
\hline 0.5 & 0.75 & -0.00656 & -0.00656 & -0.00656 & 0.74344 & 0.74344 & 0.74344 \\
\hline 0.75 & 0.875 & -0.00682 & -0.00681 & -0.00681 & 0.86818 & 0.86819 & 0.86819 \\
\hline 1 & 1 & -0.00707 & -0.00707 & -0.00707 & 0.99293 & 0.99293 & 0.99293 \\
\hline
\end{tabular}


Table 4. Comparison of results with one fluid and two fluid model for $m=k=h=1, \theta=0, \lambda x=\pi / 2, \lambda=0.05$ and $\varepsilon=0.02$.

\begin{tabular}{|c|c|c|c|c|c|c|c|c|}
\hline \multirow[b]{2}{*}{$y$} & \multicolumn{2}{|c|}{$\begin{array}{c}\text { Umavathi and Shekar } \\
\text { (2011) (Two-fluid } \\
\text { model) } h=0.1\end{array}$} & \multicolumn{2}{|c|}{$\begin{array}{l}\text { Present model } \\
\qquad h=0.1\end{array}$} & \multicolumn{2}{|c|}{$\begin{array}{c}\text { Vajravelu and } \\
\text { Sastri (1978) } \\
\text { (One-fluid model) }\end{array}$} & \multicolumn{2}{|c|}{$\begin{array}{c}\text { Present model } \\
m=k=h=1\end{array}$} \\
\hline & $u$ & $T$ & $u$ & $T$ & $u$ & $T$ & $u$ & $T$ \\
\hline-1 & 0 & 0 & 0 & 0 & 0 & 0 & 0 & 0 \\
\hline-0.75 & 0.2173 & 0.22749 & 0.2173 & 0.22749 & 0.41016 & 0.125 & 0.41014 & 0.125 \\
\hline-0.5 & 0.36363 & 0.45497 & 0.36363 & 0.45497 & 0.78125 & 0.25 & 0.78123 & 0.25 \\
\hline-0.25 & 0.36791 & 0.68243 & 0.36791 & 0.68243 & 1.07422 & 0.375 & 1.07419 & 0.375 \\
\hline 0 & 0.1591 & 0.90986 & 0.1591 & 0.90986 & 1.25 & 0.5 & 1.24998 & 0.5 \\
\hline 0 & 0.01591 & 0.90986 & 0.01591 & 0.90986 & 1.25 & 0.5 & 1.24998 & 0.5 \\
\hline 0.25 & 0.01238 & 0.93239 & 0.01238 & 0.93239 & 1.26953 & 0.625 & 1.26951 & 0.625 \\
\hline 0.5 & 0.00855 & 0.95493 & 0.00855 & 0.95493 & 1.09375 & 0.75 & 1.09374 & 0.75 \\
\hline 0.75 & 0.00443 & 0.97746 & 0.00443 & 0.97746 & 0.68359 & 0.875 & 0.68359 & 0.875 \\
\hline 1 & 0 & 1 & 0 & 1 & 0 & 1 & 0 & 1 \\
\hline
\end{tabular}

\section{Conclusions}

Mixed convection flow and heat transfer in a vertical wavy channel filled with porous and fluid layers was studied. Results were presented for variations of Grashof number, viscosity ratio, width ratio, conductivity ratio and traveling thermal temperature on the velocity, temperature, skin friction and Nusselt number.

1. Grashof number and width ratio were enhances the velocity parallel to the flow and diminishes the velocity perpendicular to the flow.

2. Viscosity ratio and conductivity ratio was to suppress the velocity parallel to the flow and promotes the velocity perpendicular to the flow.

3. The effect of Grashof number and viscosity ratio were not operative on temperature whereas, width ratio and conductivity ratio reduces the temperature.

4. It was also observed in all the results that, as the porous parameter increases, the velocity parallel to the flow decreases and increases the velocity perpendicular to the flow whereas, it remains invariant on temperature.

5. The effect of traveling thermal temperature was to enhance the velocity and temperature near the left wavy wall and remains invariant at the right wavy wall.

6. The skin friction increases at the left wall and decreases at the right wall as the Grashof number increases whereas, width ratio and porous parameter decreases the skin friction at the left wall and increases at the right wall.

7. The Nusselt number remains invariant on Grashof number whereas, the Nusselt number decreases at the left wall and increases at the right wall as the width ratio increases. Amplitude and porous parameter decreases the Nusselt number at both the walls.

8. The results obtained were in good agreement with the results for two-fluid model (Umavathi and Shekar, 2011) and for one-fluid model (Vajravelu and Sastri, 1978).

\section{Nomenclature}

a amplitude $(\mathrm{m})$

$C_{p} \quad$ specific heat at constant pressure $\left(\mathrm{kJ} \mathrm{kg}^{-1} \mathrm{~K}^{-1}\right)$

$g \quad$ acceleration due to gravity $\left(\mathrm{ms}^{-2}\right)$

Gr Grashof number $\left(h^{(1)^{3}} g \beta \Delta T / v^{2}\right)$

$h \quad$ width ratio of the channel $\left(h^{(2)} / h^{(1)}\right)$

$K \quad$ thermal conductivity $\left(\mathrm{W} \mathrm{m}^{-1} \mathrm{~K}^{-1}\right)$

$K_{e f f} \quad$ effective thermal conductivity $\left(\mathrm{W} \mathrm{m}^{-1} \mathrm{~K}^{-1}\right)$

$k \quad$ thermal conductivity ratio $\left(K_{\text {eff }} / K\right)$

$m \quad$ viscosity ratio $\left(\mu_{\text {eff }} / \mu\right)$

$\mathrm{Nu} \quad$ Nusselt number 


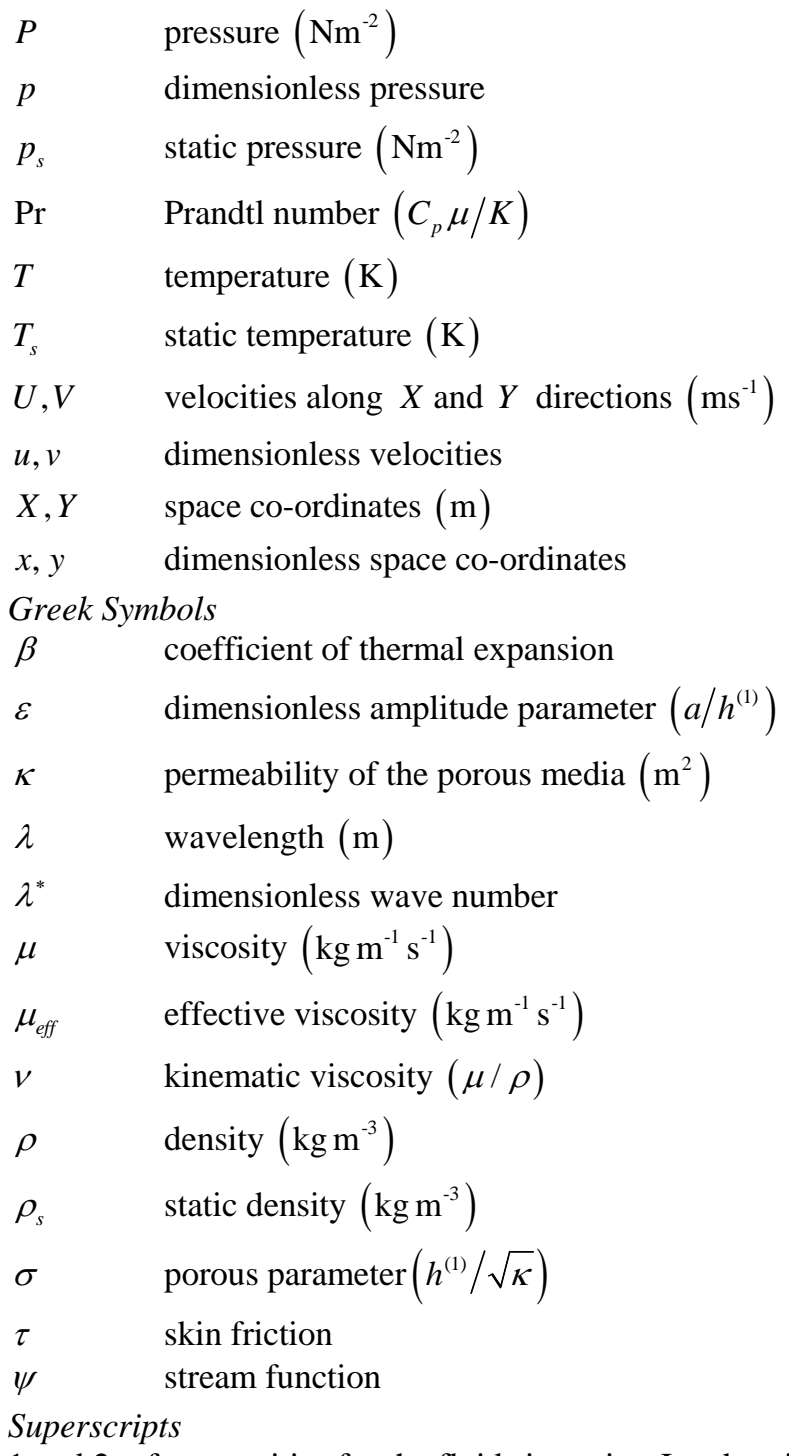

1 and 2 refer quantities for the fluids in region-I and region-II respectively.

Subscripts

p porous

fluid

0 and 1 refer quantities for the zeroth order and first order equations.

\section{Appendix}

A. Primary categories of fluid flow interface conditions between a porous medium and a fluid layer.

Model 1: $u_{p}=u_{f} ;\left(\frac{d u}{d y}\right)_{p}=\left(\frac{d u}{d y}\right)_{f}$ used by Neale and Nader (1974), Vafai and Kim (1990), Jang and Chen (1992).

Model 2: $u_{p}=u_{f} ; \mu_{e f f}\left(\frac{d u}{d y}\right)_{p}=\mu\left(\frac{d u}{d y}\right)_{f}$ used by Kim and Choi (1996), Poulikakos and Kazmierczak (1987).

Model 3: $u_{p}=u_{f} ; \frac{\mu}{\varepsilon}\left(\frac{d u}{d y}\right)_{p}-\mu\left(\frac{d u}{d y}\right)_{f}=\beta_{1} \frac{\mu}{\sqrt{K}} u$ used by Ochoa-Tapia and Whitaker (1995), Kuznetsov (1999).

Model 4: $u_{p}=u_{f} ; \frac{\mu}{\varepsilon}\left(\frac{d u}{d y}\right)_{p}-\mu\left(\frac{d u}{d y}\right)_{f}=\beta_{1} \frac{\mu}{\sqrt{K}} u+\beta_{2} \rho u^{2}$ used by Ochoa-Tapia and Whitaker (1998) . 
Model 5: $\quad\left(\frac{d u}{d y}\right)_{f}=\frac{\alpha^{*}}{\sqrt{K}}\left(u_{\text {int }}-u_{\infty}\right)$ used by Beavers and Joseph (1967), Sahraoui and Kaviany (1992). A Forchheimer term is added to the momentum equation in the porous side for the purpose of comparison.

B. Primary categories of heat transfer interface conditions between a porous medium and a fluid layer.

Model 1: $T_{p}=T_{f} ; K_{e f f} \frac{\partial T_{f}}{\partial y}=K_{f} \frac{\partial T_{p}}{\partial y}$ used by Kuznetsov (1999), Jang and Chen (1992).

Model 2: $T_{p}=T_{f} ; \phi+K_{f}\left(\frac{\partial T}{\partial y}\right)_{f}=K_{e f f}\left(\frac{\partial T}{\partial y}\right)_{p}$ used by Ochoa-Tapia and Whitaker (1998).

Model 3: $\left(\frac{d T}{d y}\right)_{p}=\frac{\alpha_{T}}{\lambda}\left(T_{p}-T_{f}\right) ; K_{\text {eff }} \frac{\partial T_{f}}{\partial y}=K_{f} \frac{\partial T_{p}}{\partial y}$ used by Sahraoui and Kaviany (1994), using fluid flow of Model 1.

Model 4: $\left(\frac{d T}{d y}\right)_{p}=\frac{\alpha_{T}}{\lambda}\left(T_{p}-T_{f}\right) ; K_{\text {eff }} \frac{\partial T_{f}}{\partial y}=K_{f} \frac{\partial T_{p}}{\partial y}$ used by Sahraoui and Kaviany (1994), using fluid flow of Model 3.

For the present problem Model A2 is used for fluid flow and Model B1 is used for heat transfer along with the continuity of pressure gradient along the flow direction.

\section{Solutions and constants}

$$
\begin{aligned}
& \theta_{0}^{(1)}=c_{1} y+c_{2}, \theta_{0}^{(2)}=c_{3} y+c_{4}, u_{0}^{(1)}=l_{1} y+l_{2}+d_{1} \cosh (\sqrt{a} y)+d_{2} \sinh (\sqrt{a} y), u_{0}^{(2)}=l_{3} y^{3}+l_{4} y^{2}+d_{3} y+d_{4}, \\
& u_{1}^{(1)}=-\cos (\lambda x)\left(2 l_{5} y+d_{6}+\sqrt{a} d_{7} \sinh (\sqrt{a} y)+\sqrt{a} d_{8} \cosh (\sqrt{a} y)\right)+\lambda \sin (\lambda x)\left(2 f_{11} y+3 f_{12} y^{2}+4 f_{13} y^{3}+5 f_{14} y^{4}\right. \\
& +\left(\sqrt{a} f_{16}+2 f_{17}\right) y \cosh (\sqrt{a} y)+\left(\sqrt{a} f_{15}+2 f_{18}\right) y \sinh (\sqrt{a} y)+\left(\sqrt{a} f_{18}+3 f_{19}\right) y^{2} \cosh (\sqrt{a} y)+\left(\sqrt{a} f_{17}+3 f_{20}\right) y^{2} \sinh (\sqrt{a} y) \text {, } \\
& +f_{15} \cosh (\sqrt{a} y)+f_{16} \sinh (\sqrt{a} y)+f_{19} \sqrt{a} y^{3} \sinh (\sqrt{a} y)+f_{20} \sqrt{a} y^{3} \cosh (\sqrt{a} y)+d_{14}+d_{15} \sqrt{a} \sinh (\sqrt{a} y) \\
& \left.+d_{16} \sqrt{a} \cosh (\sqrt{a} y)-G r c_{9} y / m a\right) \\
& u_{1}^{(2)}=-\cos (\lambda x)\left(4 l_{6} y^{3}+\frac{d_{9}}{2} y^{2}+d_{10} y+d_{11}\right)+\lambda \sin (\lambda x)\left(9 l_{27} y^{8}+8 l_{28} y^{7}+7 l_{29} y^{6}+6 l_{30} y^{5}+5 l_{31} y^{4}+4 l_{32} y^{3}+3 l_{33} y^{2}+\frac{d_{17}}{2} y\right. \text {, } \\
& \left.{ }^{2}+d_{18} y+d_{19}\right) \\
& v_{1}^{(1)}=-\lambda \sin (\lambda x)\left(l_{5} y^{2}+d_{5}+d_{6} y+d_{7} \cosh (\sqrt{a} y)+d_{8} \sinh (\sqrt{a} y)\right)-\lambda^{2} \cos (\lambda x)\left(f_{11} y^{2}+f_{12} y^{3}+f_{13} y^{4}+f_{14} y^{5}+f_{15} y \cosh (\sqrt{a} y)\right. \\
& +f_{16} y \sinh (\sqrt{a} y)+f_{17} y^{2} \cosh (\sqrt{a} y)+f_{18} y^{2} \sinh (\sqrt{a} y)+f_{19} y^{3} \cosh (\sqrt{a} y)+f_{20} y^{3} \sinh (\sqrt{a} y)-G r c_{9} /(2 m a)+d_{13}+d_{14} y \\
& \left.+d_{15} \cosh (\sqrt{a} y)+d_{16} \sinh (\sqrt{a} y)\right) \\
& v_{1}^{(2)}=-\lambda \sin (\lambda x)\left(l_{6} y^{4}+\frac{d_{9}}{6} y^{3}+\frac{d_{10}}{2} y^{2}+d_{11} y+d_{12}\right)-\lambda^{2} \cos (\lambda x)\left(l_{27} y^{9}+l_{28} y^{8}+l_{29} y^{7}+l_{30} y^{6}+l_{31} y^{5}+l_{32} y^{4}+l_{33} y^{3}+\frac{d_{17}}{6} y^{3}\right. \\
& \left.+\frac{d_{18}}{2} y^{2}+d_{19} y+d_{20}\right) \\
& \theta_{1}^{(1)}=\cos (\lambda x)\left(c_{5} y+c_{6}\right)-\lambda \sin (\lambda x)\left(l_{14} y^{6}+l_{15} y^{5}+l_{16} \cosh (\sqrt{a} y)+l_{17} \sinh (\sqrt{a} y)+l_{18} y^{4}+l_{19} y \cosh (\sqrt{a} y)+l_{20} y \sinh (\sqrt{a} y)\right. \text {, } \\
& \left.+c_{9} y+c_{10}\right) \\
& \theta_{1}^{(2)}=\cos (\lambda x)\left(c_{7} y+c_{8}\right)-\lambda \sin (\lambda x)\left(l_{21} y^{6}+l_{22} y^{5}+l_{23} y^{4}+l_{24} y^{3}+l_{25} y^{2}+c_{11} y+c_{12}\right), \\
& \tau_{-1}^{0}=l_{1}-\sqrt{a} d_{1} \cosh (\sqrt{a})+d_{2} \sqrt{a} \sinh (\sqrt{a}), \tau_{1}^{0}=3 l_{3}+2 l_{4}+d_{3}, \\
& \tau_{-1}=l_{1}-\sqrt{a} d_{1} \sinh (\sqrt{a})+d_{2} \sqrt{a} \cosh (\sqrt{a})+\varepsilon \cos (\lambda x)\left(a d_{1} \cosh (\sqrt{a})+d_{2} a \sinh (\sqrt{a})-2 l_{5}+a d_{7} \cosh (\sqrt{a})+d_{8} a \sinh (\sqrt{a})\right) \\
& +\varepsilon \lambda \sin (\lambda x)\left(2 f_{11}-6 f_{12}+12 f_{13}-20 f_{14}+\left(2 \sqrt{a} f_{16}+2 f_{17}-a f_{15}+a f_{17}-4 \sqrt{a} f_{18}-6 f_{19}-a f_{19}+6 f_{20} \sqrt{a}\right) \cosh (\sqrt{a})\right. \\
& \left.+\left(2 \sqrt{a} f_{15}+a f_{16}+4 \sqrt{a} f_{17}-2 f_{18}-a f_{18}-6 \sqrt{a} f_{19}+6 f_{20}+a f_{20}\right) \sinh (\sqrt{a})-G r c_{9} /(m a)+a d_{15} \cosh (\sqrt{a})-a d_{16} \sqrt{a} \sinh (\sqrt{a})\right)
\end{aligned}
$$


$\tau_{1}=3 l_{3}+2 l_{4}+d_{3}-\varepsilon \cos (\lambda x)\left(12 l_{6}+d_{9}+d_{10}\right)-\varepsilon \lambda \sin (\lambda x)\left(72 l_{27}+56 l_{28}+42 l_{29}+30 l_{30}+20 l_{31}+12 l_{32}+6 l_{33}+d_{17}+d_{18}\right)$, $N u_{-1}=c_{1}+\varepsilon \cos (\lambda x) c_{5}-\varepsilon \lambda \sin (\lambda x)\left(-2 l_{14}+3 l_{15}-\left(\sqrt{a}\left(l_{16}-l_{19}\right)+l_{20}\right) \sinh (\sqrt{a})+\left(\sqrt{a} l_{17}+l_{19}-\sqrt{a} l_{20}\right) \cosh (\sqrt{a})-4 l_{18}+c_{9}\right)$, $N u_{1}=c_{3}+\varepsilon \cos (\lambda x) c_{7}-\varepsilon \lambda \sin (\lambda x)\left(6 l_{21}+5 l_{22}+4 l_{23}+3 l_{24}+2 l_{25}+c_{11}\right)$.

$a=\frac{\sigma^{2}}{m}, \quad c_{3}=\frac{k h}{k h+1}, c_{1}=\frac{c_{3}}{k h}, c_{4}=1-c_{3}, \quad c_{2}=c_{4}, \quad l_{1}=\frac{G r c_{1}}{m a}, \quad l_{2}=\frac{G r c_{2}}{m a}, \quad l_{3}=-\frac{G r h^{3} c_{3}}{6}, \quad l_{4}=-\frac{G r h^{3} c_{4}}{2}, \quad l_{5}=\frac{G r c_{5}}{2 m a}$, $l_{6}=\frac{G r h^{3} c_{7}}{24}, d_{1}=\frac{d_{2} \sinh (\sqrt{a})+l_{1}-l_{2}}{\cosh (\sqrt{a})}, d_{2}=\frac{\left(l_{2}-l_{1}\right) h-\left(l_{3}+l_{4}+m h^{2} l_{1}+h l_{2}\right) \cosh (\sqrt{a})}{m h^{2} \sqrt{a} \cosh (\sqrt{a})+h \sinh (\sqrt{a})}, d_{3}=m h^{2}\left(d_{2} \sqrt{a}+l_{1}\right), c_{8}=-c_{7}-\frac{c_{3}}{h}$, $d_{4}=h\left(d_{1}+l_{2}\right), \quad c_{7}=\frac{k\left(c_{1} h \cos (\theta)-c_{3}\right)}{k h+1}, \quad c_{5}=\frac{c_{7}}{k h}, \quad c_{6}=c_{8}, \quad l_{7}=c_{1} d_{5}+c_{6} l_{2}, \quad l_{8}=c_{5} l_{2}+c_{6} l_{1}+d_{6} c_{1}, \quad l_{9}=c_{6} d_{1}+d_{7} c_{1}$, $l_{10}=c_{6} d_{2}+d_{8} c_{1}, \quad l_{11}=c_{5} l_{1}+c_{1} l_{5}, \quad l_{12}=d_{1} c_{5}, \quad l_{13}=d_{2} c_{5}, \quad l_{14}=\frac{\operatorname{Pr} l_{7}}{2 k}, \quad l_{15}=\frac{\operatorname{Pr} l_{8}}{2 k}, \quad l_{16}=\frac{\operatorname{Pr}}{k}\left(\frac{l_{9}}{a}-\frac{l_{12}}{\sqrt{a^{3}}}-\frac{l_{13}}{\sqrt{a^{3}}}\right), \quad l_{18}=\frac{\operatorname{Pr} l_{11}}{2 k}$, $l_{17}=\frac{\operatorname{Pr}}{k}\left(\frac{l_{10}}{a}-\frac{l_{12}}{\sqrt{a^{3}}}-\frac{l_{13}}{\sqrt{a^{3}}}\right), \quad l_{19}=\frac{\operatorname{Pr} l_{12}}{k a}, \quad l_{20}=\frac{\operatorname{Pr} l_{13}}{k a}, \quad l_{21}=\frac{\operatorname{Pr}}{30}\left(c_{7} l_{3}+c_{3} l_{6}\right), \quad l_{22}=\frac{\operatorname{Pr}}{20}\left(c_{7} l_{4}+c_{8} l_{3}+\frac{c_{3} d_{9}}{6}\right)$, $l_{23}=\frac{\operatorname{Pr}}{12}\left(c_{7} d_{3}+c_{8} l_{4}+\frac{c_{3} d_{10}}{2}\right), \quad l_{24}=\frac{\operatorname{Pr}}{6}\left(c_{7} d_{4}+c_{8} d_{3}+c_{3} d_{11}\right), \quad l_{25}=\frac{\operatorname{Pr}}{2}\left(c_{8} d_{4}+c_{3} d_{12}\right), \quad f_{1}=2 l_{1} l_{5}+2 G r l_{14}, \quad f_{2}=3 G r l_{15}$, $f_{3}=4 G r l_{18}, \quad f_{4}=a l_{2} d_{7}+2 l_{5} d_{1}-a d_{1} d_{5}+G r\left(l_{17} \sqrt{a}+l_{19}\right), \quad f_{9}=-a d_{2} l_{5}, \quad f_{5}=a l_{2} d_{8}+2 l_{5} d_{2}-a d_{2} d_{5}+G r\left(l_{16} \sqrt{a}+l_{20}\right)$, $f_{6}=a l_{1} d_{7}-a d_{1} d_{6}+G r l_{20} \sqrt{a}, \quad f_{7}=a l_{1} d_{8}-a d_{2} d_{6}+G r l_{19} \sqrt{a}, \quad f_{8}=-a d_{1} l_{5}, \quad f_{10}=2 l_{2} l_{5}, \quad f_{11}=-\frac{f_{2}}{m a^{2}}-\frac{f_{10}}{2 m a}$, $f_{12}=-\frac{f_{1}}{6 m a}-\frac{f_{3}}{m a^{2}}, \quad f_{13}=-\frac{f_{2}}{12 m a}, \quad f_{14}=-\frac{f_{3}}{20 m a}, \quad f_{15}=\frac{f_{5}}{2 m \sqrt{a^{3}}}-\frac{5 f_{6}}{4 m a^{2}}+\frac{17 f_{9}}{4 m \sqrt{a^{5}}}, \quad f_{16}=\frac{f_{4}}{2 m \sqrt{a^{3}}}-\frac{5 f_{7}}{4 m a^{2}}+\frac{17 f_{8}}{4 m \sqrt{a^{5}}}$, $f_{17}=\frac{f_{7}}{4 m \sqrt{a^{3}}}-\frac{5 f_{8}}{4 m a^{2}}, \quad f_{18}=\frac{f_{6}}{4 m \sqrt{a^{3}}}-\frac{5 f_{9}}{4 m a^{2}}, \quad f_{19}=\frac{f_{9}}{6 m \sqrt{a^{3}}}, \quad f_{20}=\frac{f_{8}}{6 m \sqrt{a^{3}}}, \quad f_{21}=6 l_{3} l_{6}+6 G r h^{3} l_{21}, \quad f_{22}=10 l_{4} l_{6}+5 G r h^{3} l_{22}$, $f_{23}=12 d_{3} l_{6}+\frac{2 l_{4} d_{9}}{3}-2 l_{3} d_{10}+4 G r h^{3} l_{23}, \quad f_{24}=12 d_{4} l_{6}+d_{3} d_{9}-6 l_{3} d_{11}+3 G r h^{3} l_{24}, \quad f_{25}=d_{4} d_{9}+d_{3} d_{10}-6 l_{3} d_{12}-2 l_{4} d_{11}+2 G r h^{3} l_{25}$, $f_{26}=d_{4} d_{10}-2 l_{4} d_{12}, \quad f_{27}=\frac{f_{21}}{3024}, \quad f_{28}=\frac{f_{22}}{1680}, \quad f_{29}=\frac{f_{23}}{840}, \quad f_{30}=\frac{f_{24}}{360}, \quad f_{31}=\frac{f_{25}}{120}, \quad f_{32}=\frac{f_{26}}{24}, \quad f_{33}=\frac{G r h^{3} c_{11}}{6}$; $z_{1}=\cos (\theta)\left(-d_{1} \sqrt{a} \sinh (\sqrt{a})+d_{2} \sqrt{a} \cosh (\sqrt{a})+l_{1}\right)+2 l_{5}, \quad z_{2}=\frac{3 l_{3}+2 l_{4}+d_{3}}{h}-4 l_{6}, \quad z_{3}=\frac{-m a h^{3}+6 h}{6} \quad z_{4}=\frac{m a h^{2}+2 h}{2}$, $z_{5}=l_{6}+m h^{2} l_{5}, \quad z_{6}=\frac{-m a h^{3}+2 h}{2}, z_{7}=m h^{2} a, z_{8}=2 m h^{2} l_{5}-z_{2}, \quad z_{9}=h+z_{3}, z_{10}=z_{4}-h \cosh (\sqrt{a}), z_{11}=h \sqrt{a}+h \sinh (\sqrt{a})$, $z_{12}=-h l_{5}+z_{5}, \quad z_{13}=z_{7}+z_{6} \sqrt{a} \sinh (\sqrt{a}), \quad z_{14}=h \sqrt{a}-z_{6} \sqrt{a} \cosh (\sqrt{a}), \quad z_{15}=z_{8}+z_{1} z_{6}, \quad z_{16}=z_{10}+z_{9} \sqrt{a} \sinh (\sqrt{a})$, $z_{17}=z_{11}-z_{9} \sqrt{a} \cosh (\sqrt{a}), z_{18}=z_{12}+z_{1} z_{9} ; d_{8}=\frac{z_{13} z_{18}-z_{15} z_{16}}{z_{16} z_{14}-z_{13} z_{17}}, d_{7}=\frac{-z_{15}-z_{14} d_{8}}{z_{13}}, d_{6}=d_{7} \sqrt{a} \sinh (\sqrt{a})-d_{8} \sqrt{a} \cosh (\sqrt{a})+z_{1}$, $d_{5}=d_{8} \sinh (\sqrt{a})-d_{7} \cosh (\sqrt{a})+d_{6}-l_{5}, \quad d_{9}=-h^{3} \operatorname{mad}_{6}, \quad d_{10}=m h^{2}\left(2 l_{5}+a d_{7}\right), \quad d_{11}=h\left(d_{6}+\sqrt{a} d_{8}\right), \quad d_{12}=h\left(d_{5}+d_{7}\right) ;$ $z_{19}=l_{14}-l_{15}+\left(l_{16}-l_{19}\right) \cosh (\sqrt{a})+\left(l_{20}-l_{17}\right) \sinh (\sqrt{a})+l_{18}, \quad z_{20}=l_{21}+l_{22}+l_{23}+l_{24}+l_{25}, \quad z_{21}=k h\left(c_{6}+l_{17} \sqrt{a}+l_{19}-\frac{c_{8}}{k h}\right)$; $c_{9}=\frac{z_{19}-z_{20}-z_{21}-l_{16}}{k h+1}, \quad c_{10}=c_{9}-z_{19}, \quad c_{11}=z_{21}+k h c_{9}, \quad c_{12}=l_{16}+c_{10}, \quad z_{24}=f_{27}+f_{28}+f_{29}+f_{30}+f_{31}+f_{32}$, $z_{22}=f_{11}-f_{12}+f_{13}-f_{14}\left(-f_{15}+f_{17}-f_{19}\right) \cosh (\sqrt{a})+\left(f_{16}-f_{18}+f_{20}\right) \sinh (\sqrt{a}), \quad z_{23}=z_{231}+z_{232}, \quad z_{27}=z_{271}+z_{272}$, $z_{231}=-2 f_{11}+3 f_{12}-4 f_{13}+5 f_{14}+\left(f_{15}-\sqrt{a} f_{16}-2 f_{17}+\sqrt{a} f_{18}+3 f_{19}-\sqrt{a} f_{20}\right) \cosh (\sqrt{a}), \quad z_{25}=9 f_{27}+8 f_{28}+7 f_{29}+6 f_{30}+5 f_{31}+4 f_{32}$ $z_{232}=\left(\sqrt{a} f_{15}-f_{16}-\sqrt{a} f_{17}+2 f_{18}+\sqrt{a} f_{19}-3 f_{20}\right) \sinh (\sqrt{a}), \quad z_{26}=2 m h^{2}\left(f_{11}+\sqrt{a} f_{16}+f_{17}-\frac{2 G r c_{9}}{m a}\right), \quad z_{28}=\frac{1}{6}\left(-h^{3} m a+6 h\right)$, 


$$
\begin{aligned}
& z_{271}=-\left(d_{1}+l_{2}\right)\left(d_{6}+\sqrt{a} d_{8}\right)+\left(l_{1}+\sqrt{a} d_{2}\right)\left(d_{5}+d_{7}\right), \quad z_{272}=m\left(6 f_{12}+3 a f_{15}+6 \sqrt{a} f_{18}+6 f_{19}\right)-\sigma^{2} f_{15}-G r l_{16}+d_{4} d_{11}-d_{3} d_{12}, \\
& z_{29}=\frac{1}{2}\left(h^{2} m a+2 h\right), \quad z_{30}=z_{24}+\frac{z_{26}}{2}+\frac{z_{27}}{6}+h f_{15}, \quad z_{31}=\frac{1}{2}\left(-h^{3} m a+2 h\right), \quad z_{32}=h^{2} m a, z_{33}=z_{25}+z_{26}+\frac{z_{27}}{2}+h f_{15}, \quad z_{34}=z_{28}+h, \\
& z_{35}=z_{29}-h \cosh (\sqrt{a}), \quad z_{36}=h \sqrt{a}+h \sinh (\sqrt{a}), \quad z_{37}=z_{30}-h z_{22}, \quad z_{38}=z_{32}+z_{31} \sqrt{a} \sinh (\sqrt{a}), \quad z_{39}=h \sqrt{a}-z_{31} \sqrt{a} \cosh (\sqrt{a}), \\
& z_{40}=z_{33}-z_{23} z_{31}, \quad z_{41}=z_{35}+z_{34} \sqrt{a} \sinh (\sqrt{a}), \quad z_{42}=z_{36}-z_{34} \sqrt{a} \cosh (\sqrt{a}), \quad z_{43}=z_{37}-z_{23} z_{34}, \quad d_{15}=\frac{-z_{39} d_{16}+z_{40}}{z_{38}}, \\
& d_{13}=d_{14}-d_{15} \cosh (\sqrt{a})+d_{16} \sinh (\sqrt{a})-z_{22}, \quad d_{14}=d_{15} \sqrt{a} \sinh (\sqrt{a})-d_{16} \sqrt{a} \cosh (\sqrt{a})-z_{23}, \quad d_{16}=\frac{z_{38} z_{43}-z_{40} z_{41}}{z_{30} z_{41}-z_{38} z_{42}}, \\
& d_{17}=-m a h^{3} d_{14}+z_{27}, d_{18}=m a h^{2} d_{15}+z_{26}, d_{19}=h\left(d_{14}+\sqrt{a} d_{16}+f_{15}\right), d_{20}=h\left(d_{13}+d_{15}\right) .
\end{aligned}
$$

\section{Acknowledgements}

The authors would like to thank UGC-New Delhi for the financial support under UGC-Major Research Project.

\section{References}

Alazmi, M. and Vafai, K., 2001. Analysis of fluid flow and heat transfer interfacial conditions between a porous medium and a fluid layer, Int. J. Heat Mass Transfer, Vol. 44, pp. 1735-1749.

Al-Nimr, M.A. and Alkam, M.K., 1998. Unsteady non -Darcian fluid flow in a parallel channels filled with porous material, Heat Mass Transfer, Vol. 33, pp. 315-318.

Beavers, G.S. and Joseph, D.D., 1967. Boundary conditions at naturally permeable wall, J. Fluid Mech., Vol. 13, pp. 197-207.

Chikh, S., Boumedian, A., Bouhadef, K. and Lauriat, G., 1995. Analytical solution of non-Darcian forced convection in an annular duct partially filled with porous medium, Int. J. Heat Mass Transfer, Vol. 38, pp. 1543-1551.

Deajani, G., Talsim, M.E. and Narusawa, U., 1986. Effects of boundary conditions on thermal instability of superposed porous and fluid layer, Natural Convection in Enclosures, R.S. Figliola and I. Catton (eds.), ASME, New York, pp. 83-89

Eldabe, N.T.M., El-Sayed, M.F., Ghaly, A.Y. and Sayed, H.M., 2008. Mixed convective heat and mass transfer in a nonNewtonian fluid at a peristaltic surface with temperature dependent viscosity, Arch, Appl. Mech., Vol. 78, pp. 599-624.

Gilver, R.C. and Altobelli, S.A., 1994. A determination of the effective viscosity for Brinkman-Forchheimer flow model, J. Fluid Mech., Vol. 258, pp. 355-370.

Ingham, D.B. and Pop, I., 2005. Transport phenomena in porous media, Elsevier, Oxford.

Jang, J.Y. and Chen, J.L., 1992. Forced convection in a parallel plate channel partially filled with a high porosity medium, Int. Commun. Heat Mass Transfer, Vol. 19, pp. 263-273.

Jang, J.H., Yan, W.M. and Liu, H.C., (2003). Natural convection heat and mass transfer along a vertical wavy surface, Int. J. Heat Mass Transfer, Vol. 46, pp. 1075-1083.

Jang, J.H. and Yan, W.M., 2004. Mixed convection heat and mass transfer along a vertical wavy surface, Int. J. Heat Mass Transfer, Vol. 47, pp. 419-428.

Kim, S.J. and Choi, C.Y., 1996. Convection heat transfer in porous and overlying layers heated from below, Int. J. Heat Mass Transfer, Vol. 39, pp. 319-329.

Kuznetsov, A.V., 1999. Fluid mechanics and heat transfer in the interface region between a porous medium and a fluid layer: a Boundary layer solution, J. Porous Media, Vol. 2(3), pp. 309-321.

Kuznetsov, A.V., 1998. Analytical investigation of Couette flow in a composite channel partially filled with porous medium and partially filled with clear fluid, Int. J. Heat Mass Transfer, Vol. 41, pp. 2556-2560.

Li Tang, Di Liu, Fu-Yun Zhao, Guang-Fa Tang, 2010. Combined heat and moisture convective transport in a partial enclosure with multiple free ports, Applied Thermal Engineering, Vol. 30, pp. 977-990.

Luo, H., Blyth, M.G. and Pozrikidis, C., 2008. Two-layer flow in a corrugated channel, J. Eng. Math. Vol. 60, pp.127-147.

Malashetty, M.S., Umavathi, J.C. and Leela, V., 2001. Magnetoconvective flow and heat transfer between vertical wavy wall and a parallel flat wall, Int. J. Appl. Mech. Engg., Vol. 6(2), pp. 437-456.

Malashetty, M.S., Umavathi, J.C. and Prathap Kumar, J., 2004. Two fluid flow and heat transfer in an inclined channel containing porous and fluid layer, Heat and Mass Transfer, Vol. 40, pp. 871-876.

Malashetty, M.S., Umavathi, J.C. and Prathap Kumar, J., 2005. Flow and heat transfer in an inclined channel containing a fluid layer sandwiched between two porous layers, J. Porous Media, Vol. 8, No. 5, pp. 443-453.

Martys, N., Bentz, D.P. and Garboczi, E.J., 1994. Computer simulation study of the effective viscosity in Brinkman's equation, Phys. Fluids, Vol. 6, pp. 1434-1439.

Masuoka, T., 1974. Convective currents in a horizontal layer divided by a permeable wall, Bull. Japan, Soc. Mech. Eng., Vol. 17, pp. 225-237.

Neale, G. and Nader, W., 1974. Practical significance of Brinkman's extension of Darcy's law: coupled parallel flows within a channel and a bounding porous medium, Can. J. Chem. Engrg., Vol. 52, pp. 475-478. 
Neild, D.A., 1991. The limitations of Brinkman-Forchhiemer equation in modeling flow in a saturated porous medium and at an interface, Int. J. Heat Mass Transfer, Vol. 12, pp. 269-272.

Nield, D.A., 1983. The boundary correction for the Rayleigh-Darcy problem: Limitations of Brinkman equation, J. Fluid Mech., Vol. 128, pp. 37-46.

Nield, D.A., and Bejan, A., 2006. Convection in porous media (3rd Ed.), Springer-Verlag, New York.

Ochoa-Tapia, J.A. and Whitaker, S., 1995. Momentum transfer at the boundary between a porous medium and homogeneous fluid I: Theoretical development, Int. J. Heat Mass Transfer, Vol. 38, pp. 2635-2646.

Ochoa-Tapia, J.A. and Whitaker, S., 1998. Heat transfer at the boundary between a porous medium and a homogeneous fluid: the one-equation model, J. Porous Media, Vol. 1, pp. 31-46.

Prathap Kumar, J., Umavathi, J.C., Pop, I. and Basavaraj M Biradar, 2009. Full developed mixed convection flow in a vertical channel containing porous and fluid layers with isothermal or isoflux boundaries, Trans. Porous Media, Vol. 80, pp. 117-135.

Poulikakos, D. and Kazmierczak, M., 1987. Forced convective in a duct partially filled with a porous material, ASME J. Heat Mass Transfer, Vol. 109, pp. 653-662.

Rudraiah, N., 1985. Forced convection in a parallel plate channel partially filled with a porous material, ASME J. Heat Transfer, Vol. 107, pp. 331-332.

Sahraoui, M. and Kaviany, M., 1992. Slip and no-slip velocity boundary conditions at the interface of porous, plain media, Int. J. Heat Mass Transfer, Vol. 35, pp. 927-943

Sahraoui, M. and Kaviany, M., 1994. Slip and no-slip temperature boundary conditions at the interface of porous, plain media: convection, Int. J. Heat Mass Transfer, Vol. 37, pp. 1029-1044.

Srinivas, S. and Muthuraj, R., 2010. MHD flow with slip effects and temperature-dependent heat source in a vertical wavy porous space, Chem. Eng. Comm. Vol. 197, pp. 1387-1403.

Umavathi, J.C., Chamka, A.J., Abdul Mateen, and Al-Mudhaf, A., 2006. Oscillatory flow and heat transfer in horizontal composite porous medium channel, Int. Heat and Tech., Vol. 24, pp. 75-86.

Umavathi, J.C., Ali. J. Chamkha and Sridhar, K.S.R., 2010. Generalised plain Couette flow heat transfer in a composite channel, Trans Porous Media, Vol. 85, pp. 157-169.

Umavathi, J.C., Prathap Kumar, J. and Shekar, M., 2010. Mixed convective flow of immiscible viscous fluids confined between a long vertical wavy wall and a parallel flat wall, Int. J. Engg. Sci. Tech., Vol. 2, No. 6, pp. 256-277.

Umavathi, J.C. and Shekar, M., 2011. Mixed convective flow of two immiscible viscous fluids in a vertical wavy channel with traveling thermal waves, Heat Transfer Asian Research, Vol. 40, No.7, pp. 608-640.

Vafai, K. and Thiyagaraja, R., 1987. Analysis of flow and heat transfer at the interface region of a porous medium, Int. J. Heat Mass Transfer, Vol. 30, pp. 1391-1405.

Vafai, K., 2005. Handbook of porous media (2nd Ed.), Taylor \& Francis, Boca Raton.

Vafai, K. and Kim, S.J., 1990. Fluid mechanics of interface region between a porous medium and a fluid layer- an exact solution, Int. J. Heat Mass Transfer, Vol. 11, pp. 254-256.

Vafai, K. and Kim, S.J., 1995. On the limitations of the Brinkman-Forchheimer-extended Darcy equation, Int. J. Heat Mass Transfer, Vol. 16, pp. 11-15.

Vajravelu, K. and Sastri, K.S., 1978. Free convective heat transfer in a viscous incompressible fluid confined between a long vertical wavy wall and a parallel flat wall, J. Fluid Mech., Vol. 86, pp. 365-383.

Vajravelu, K, 1989. Combined free and forced convection in hydromagnetic flows in vertical wavy channels with traveling thermal waves, Int. J. Engg. Sci., Vol. 30, pp. 278-289.

Yasin Varol and Hakan F. Oztop, (2006). Free convection in a shallow wavy enclosure, Int. Commun. Heat and Mass Transfer, Vol. 33, pp. 764-771.

\section{Biographical notes}

J. C. Umavathi received Ph. D degree from Gulbarga University Gulbarga India in 1992. She is a Professor in the Department of Mathematics, Gulbarga University, Gulbarga, Karnataka, India. Her research interest includes heat and mass transfer of multiple (Newtonian and non-Newtonian) fluids through channels and rectangular ducts, numerical simulation using Finite differences and Range-Kutta Gill method, magnetohydradynamics, flow through porous media she has published more than 70 papers in referred International journals. She has also presented more than 20 research articles in National and International conferences. She is currently dealing with few projects sponsored by Government of India.

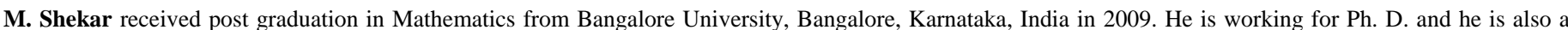

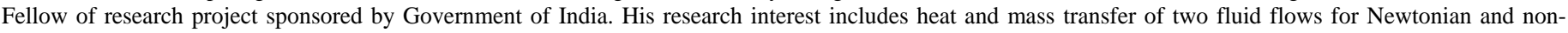
Newtonian fluids through wavy channels.

Received May 2011

Accepted November 2011

Final acceptance in revised form December 2011 